\title{
ROBINSON-SCHENSTED-KNUTH CORRESPONDENCE IN THE REPRESENTATION THEORY OF THE GENERAL LINEAR GROUP OVER A NON-ARCHIMEDEAN LOCAL FIELD
}

\author{
MAXIM GUREVICH AND EREZ LAPID, WITH AN APPENDIX BY MARK SHIMOZONO
}

ABstract. We construct new "standard modules" for the representations of general linear groups over a local non-archimedean field. The construction uses a modified Robinson-Schensted-Knuth correspondence for Zelevinsky's multisegments.

Typically, the new class categorifies the basis of Doubilet, Rota, and Stein (DRS) for matrix polynomial rings, indexed by bitableaux. Hence, our main result provides a link between the dual canonical basis (coming from quantum groups) and the DRS basis.

\section{Contents}

1. Introduction

2. RSK for multisegments

3. Relation with the Mœglin-Waldspurger involution

4. Representation theoretic applications

5. Enhanced multisegments

6. $\quad$ Odds and ends

Appendix A. Multisegments, MW, and RSK

by Mark Shimozono

Acknowledgments

\section{INTRODUCTION}

The Zelevinsky classification [33, which is the culmination of Bernstein-Zelevinsky theory, is one of the cornerstones of the representation theory of reductive groups over a non-archimedean local field $F$. It classifies the equivalence classes of the irreducible (complex, smooth) representations of the general linear groups of all ranks over $F$ in terms of multisegments, which are essentially a combinatorial object.

The irreducible representations may all be obtained as socles of the so-called standard modules (that are also indexed by multisegments). The standard modules are the parabolic induction of certain irreducible, essentially square-integrable representations, and constitute a basis for the Grothendieck group. The situation is analogous to that in category $\mathcal{O}$ where the Verma modules play the role of standard 2021.

Received by the editors June 18, 2020, and, in revised form, October 8, 2020, and May 4,

2020 Mathematics Subject Classification. Primary 05E10, 22E50.

The first author was partially supported by the Israel Science Foundation (grant No. 737/20). 
modules. This is in fact not a coincidence. It is explained by the Arakawa-Suzuki functors [1] which provide a link between category $\mathcal{O}$ of type $A$ and representations of $\mathrm{GL}_{n}(F), n \geq 0$. See [2,12,17] for more details.

In this paper we present a new class of $R S K$-standard modules that are parabolically induced from ladder representations. Its construction relies on an application of the well-known Robinson-Schensted-Knuth (RSK) correspondence. The new class is again in bijection with irreducible representations, in such a way that each irreducible representation is realized as a subrepresentation (and conjecturally, the socle) of the corresponding RSK-standard module 1

Ladder representations are a class of irreducible representations with particularly nice properties [2, 10, 14, 15]. In particular, parabolic induction from two ladder representations is well understood.

Let us describe the new construction in more detail. Roughly speaking, a multisegment $\mathfrak{m}$ is a collection of pairs of integers $\left[a_{i}, b_{i}\right], i=1, \ldots, n$, with $a_{i} \leq b_{i}$. Denote by $Z(\mathfrak{m})$ the irreducible representation of $\mathrm{GL}_{N}(F)\left(\right.$ with $N=\sum_{i}\left(b_{i}-a_{i}+1\right)$ ) corresponding to $\mathfrak{m}$, as defined by Zelevinsky.

Now, the RSK correspondence attaches to $\mathfrak{m}$ a pair of semistandard Young tableaux of the same shape of total size $n$. For our purposes it will be more convenient to use a modified version of RSK, $\mathfrak{m} \mapsto\left(P_{\mathfrak{m}}, Q_{\mathfrak{m}}\right)$ where $P_{\mathfrak{m}}$ and $Q_{\mathfrak{m}}$ are inverted Young tableaux (of the same shape, of total size $n$ ). By an inverted Young tableau, we mean that the entries in each row are strictly decreasing and the entries in each column are weakly decreasing, unlike the usual convention in which the entries in each row are weakly increasing and the entries in each column are strictly increasing. As in the classical case, $P_{\mathfrak{m}}$ is filled by the $a_{i}$ 's and $Q_{\mathfrak{m}}$ by the $b_{i}$ 's.

Suppose that the pair $\left(P_{\mathfrak{m}}, Q_{\mathfrak{m}}\right)$ is given by

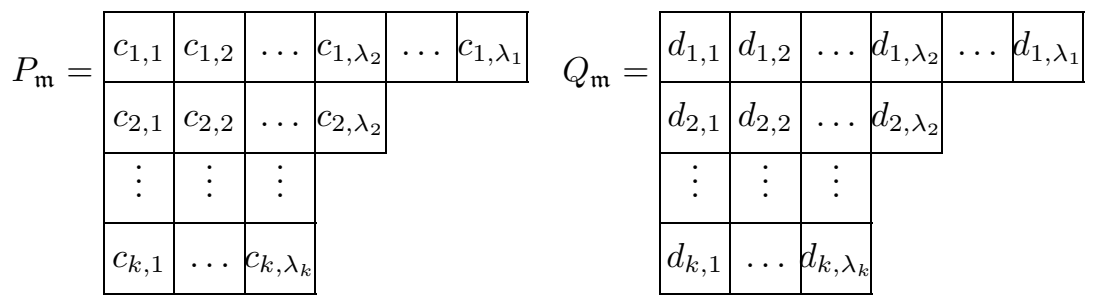

To each row of the resulting shape we attach a ladder representation by setting

$$
\pi_{i}=Z\left(\left[c_{i, 1}, d_{i, 1}\right]+\ldots+\left[c_{i, \lambda_{i}}, d_{i, \lambda_{i}}\right]\right), \quad i=1, \ldots, k .
$$

In other words, $\pi_{i}$ is the irreducible representation whose defining multisegment is encoded in the pair of $i$-th rows of the tableaux $\left(P_{\mathfrak{m}}, Q_{\mathfrak{m}}\right)$.

The RSK-standard module attached to $\mathfrak{m}$ is now defined as

$$
\Lambda(\mathfrak{m})=\pi_{k} \times \cdots \times \pi_{1},
$$

where the (Bernstein-Zelevinsky) product denotes (normalized) parabolic induction.

A different angle on the new class of modules comes from combinatorial invariant theory. Let $\mathcal{R}$ be the direct sum of Grothendieck groups of smooth finite-length representations, of all $\mathrm{GL}_{n}(F), n \geq 0$. The Bernstein-Zelevinsky product makes $\mathcal{R}$ into a commutative ring and Zelevinsky showed that $\mathcal{R}$ is the polynomial ring

\footnotetext{
${ }^{1}$ Note added in proof: Conjecture 6.1 is now proved by the first-named author (see [11).
} 
over $\mathbb{Z}$ freely generated by the classes of irreducible, essentially square-integrable representations. In other words, the standard modules form the monomial basis for $\mathcal{R}$ (as a $\mathbb{Z}$-module). On the other hand, in the context of invariant theory, Doubilet, Rota, and Stein constructed a basis for polynomial matrix rings, parameterized by pairs of tableaux, consisting of products of minors [7] (see also [6, 29]). As will be explained in 66.3 , the RSK-standard modules categorify the DRS basis for matrix polynomial rings. This phenomenon follows from the paramount determinantal property [15] of ladder representations. The construction of $\Lambda(\mathfrak{m})$ as a product of ladders parallels the recipe of DRS for obtaining all basis elements as products of minors.

Our main result is the following.

Theorem 1.1. For every multisegment $\mathfrak{m}, Z(\mathfrak{m})$ occurs as a subrepresentation of $\Lambda(\mathfrak{m})$. More precisely,

$$
Z(\mathfrak{m}) \cong \operatorname{soc}\left(\operatorname{soc}\left(\ldots \operatorname{soc}\left(\operatorname{soc}\left(\pi_{k} \times \pi_{k-1}\right) \times \pi_{k-2}\right) \times \ldots \times \pi_{2}\right) \times \pi_{1}\right) .
$$

Here, $\operatorname{soc}(\tau)$ stands for the socle (i.e., the maximal semisimple subrepresentation) of a representation $\tau$.

We expect (Conjecture 6.1) that as in Zelevinsky's case, $\operatorname{soc}(\Lambda(\mathfrak{m}))$ is itself irreducible (hence, isomorphic to $Z(\mathfrak{m})$ ) and occurs with multiplicity one in the JordanHölder sequence of $\Lambda(\mathfrak{m}) 2$

Note that the parameter $k=k(\mathfrak{m})$, i.e., the number of rows in the tableaux $P_{\mathfrak{m}}, Q_{\mathfrak{m}}$, is the width of the multisegment, as defined and studied in [10]. In particular, it was shown in [ibid.] that $k$ is the minimal number of ladder representations whose product contains $Z(\mathfrak{m})$ as a subquotient. In that respect, the RSK-standard modules possess a minimality property.

The case of Theorem 1.1 with $k(\mathfrak{m})=2$ and certain regularity conditions was previously shown in [9] using quantum shuffle methods and equivalences to module categories of quiver Hecke algebras. In fact, the papers [9, 10] were the point of departure of the present work.

Coming back to the categorification context, irreducible representations are known to give the (dual) canonical basis (à la Lusztig or Kashiwara) of $\mathcal{R}$ (see [19], for example). Thus, Theorem 1.1 gives a certain link between the DRS basis and the canonical basis. Similar links were explored in [20] by means of quantum matrix rings. Our results and expectations open a new perspective on those links in a categorial setting, i.e., it is the submodule structure which is used to characterize relations between those two bases.

A natural question which arises is what can be said about the other irreducible constituents of $\Lambda(\mathfrak{m})$. Based on empirical evidence, we conjecture that for any irreducible subquotient $Z(\mathfrak{n})$ of $\Lambda(\mathfrak{m})$ other than $Z(\mathfrak{m})$, the RSK-data $\left(P_{\mathfrak{n}}, Q_{\mathfrak{n}}\right)$ is strictly smaller than $\left(P_{\mathfrak{m}}, Q_{\mathfrak{m}}\right)$ with respect to the product order of the domination order of inverted Young tableaux (see 6.2 ). Once again, this would be analogous to the situation for Zelevinsky classification, where the pertinent partial order on multisegments (as originally defined in [33]) is closely related to the Bruhat order on the symmetric group.

In particular, this would give a positive answer to the question which still remains open, of whether the classes of RSK-standard modules indeed form a $\mathbb{Z}$-basis

\footnotetext{
${ }^{2}$ Note added in proof: Conjecture 6.1 is now proved by the first-named author (see [11]).
} 
for (an appropriate subgroup of) $\mathcal{R}$. Certain related questions can be answered (Proposition 6.5) using the basis theorem of [6].

Recall that the classical RSK correspondence admits several (equivalent) implementations. For our purposes, it is best to use Knuth's algorithm [13, §4] which constructs the Young tableaux row by row, rather than the earlier (and perhaps more commonly used) Robinson-Schensted insertion/bumping algorithm which fills them box by box. (A pictorial approach to Knuth's algorithm was given by Viennot [30.)

A key part of the proof of Theorem 1.1 is an intriguing relation between the Knuth algorithm and the description of the socles of certain induced representations due to the second-named author and Mínguez [16]. In turn, this description is closely related to the Mœglin-Waldspurger algorithm for the Zelevinsky involution [21, §II.2]. In fact, the main new combinatorial input (Corollary [3.4), which is interesting in its own right, is that roughly speaking, under certain conditions the Knuth algorithm commutes with the first step of the Mœglin-Waldspurger algorithm.

In an appendix by Mark Shimozono, further illuminating relations between those two combinatorial algorithms are presented. The slightly different point of view of the appendix suggests that some of our questions may be resolved in future work through the combinatorial theory of tableaux and crystals.

The connection between RSK and the Moglin-Waldspurger algorithm is further elucidated by considering enhanced multisegments, that is, "multisegments with dummies". More precisely, starting with a (genuine) multisegment $\mathfrak{m}=\Delta_{1}+\cdots+$ $\Delta_{k}$ we add empty (dummy) segments of the form $[a, a-1], a \in \mathbb{Z}$. This will not affect $Z(\mathfrak{m})$ but will change the output of RSK. In Theorem 5.1, to any irreducible representation $\pi=Z(\mathfrak{m})$ we concoct a whole slew of "standard modules" admitting $\pi$ as a subrepresentation (and conjecturally, as the socle) by adding to $\mathfrak{m}$ dummy segments at will.

Let $n_{a}, a \in \mathbb{Z}$ be the number of dummy segments of the form $[a, a-1]$. There are two opposite extreme cases, as stated in Proposition 5.3. If $n_{a} \ll n_{a+1}$ for all $a$ in the support of $\mathfrak{m}$, then the standard module will be the one defined by Zelevinsky (taking the product $Z\left(\Delta_{1}\right) \times \cdots \times Z\left(\Delta_{k}\right)$ in a suitable order). On the other hand, if $n_{a} \gg n_{a+1}$ for all $a$ in the support of $\mathfrak{m}$, then we will get the standard module in the sense of Langlands. In other words, the output of RSK essentially coincides (up to dummy segments) with the Mœglin-Waldspurger algorithm. Thus, we get a family of standard modules interpolating between the standard modules of Zelevinsky and Langlands.

Both the Zelevinsky classification and the RSK correspondence admit geometric interpretations (starting with 31, 32 for the former, 23, 26, 28 for the latter). However, we are not aware of a geometric interpretation of the abovementioned partial order defined through RSK. It would be interesting to find a geometric interpretation of the results and conjectures presented here.

Finally, it would be interesting to know whether other type $A$ representation categories, such as those of quiver Hecke algebras (through the Brundan-Kleshchev isomorphisms [4), quantum affine algebras (through quantum affine Schur-Weyl duality [5]) or even the classical category $\mathcal{O}$ (through the Arakawa-Suzuki functors [1,12]), can be used to reinterpret the results of this paper. 


\section{RSK FOR MULTISEGMENTS}

2.1. Multisegments. A segment $\Delta$ (of length $b-a+1$ ) is a subset of $\mathbb{Z}$ of the form

$$
[a, b]=\{n \in \mathbb{Z}: a \leq n \leq b\}
$$

for some integers $a \leq b$. We will write $b(\Delta)=a$ and $e(\Delta)=b$. We also write $\overleftarrow{\Delta}=[a-1, b-1]$ and $^{-} \Delta=[a+1, b]$. Note that if $a=b$, then ${ }^{-} \Delta$ is the empty set.

We denote by Seg the set of all segments.

Given $\Delta_{1}, \Delta_{2} \in$ Seg, we write $\Delta_{1} \ll \Delta_{2}$ if $b\left(\Delta_{1}\right)<b\left(\Delta_{2}\right)$ and $e\left(\Delta_{1}\right)<e\left(\Delta_{2}\right)$. This is a strict partial order on Seg.

A multisegment is a multiset of segments, i.e., a formal finite sum $\mathfrak{m}=\sum_{i \in I} \Delta_{i}$ where $\left\{\Delta_{i} \in \operatorname{Seg}\right\}_{i \in I}$ are segments.

If $\mathfrak{m} \neq 0$, we will write $\min \mathfrak{m}=\min _{i \in I} b\left(\Delta_{i}\right)$.

The size $|\mathfrak{m}|$ of a multisegment $\mathfrak{m}$ is, by definition, the sum $\sum_{i \in I}\left|\Delta_{i}\right|$ of the cardinalities of its segments.

For any set $X$, we denote by $\mathbb{N}(X)$ the ordered commutative monoid of finite multisets on $X$, that is, finitely supported functions from $X$ to $\mathbb{N}=\mathbb{Z}_{>0}$. We view elements of $\mathbb{N}(X)$ as formal finite (possibly empty) sums of elements of $X$.

From this point of view, we denote by $\mathfrak{M}:=\mathbb{N}(\mathrm{Seg})$ the ordered monoid of multisegments. If $\mathfrak{m}^{\prime} \leq \mathfrak{m}$, we say that $\mathfrak{m}^{\prime}$ is a sub-multisegment of $\mathfrak{m}$.

An important class of multisegments is the so-called ladders. By definition, a ladder is a nonzero multisegment of the form

$$
\mathfrak{l}=\sum_{i=0}^{k} \Delta_{i} \text { where } \Delta_{i} \ll \Delta_{i-1} \text { for } i=1, \ldots, k .
$$

Such an expression is of course unique, and we refer to it as the standard form of a ladder. We will write Lad $\subseteq \mathfrak{M}$ for the collection of all ladders.

2.2. Modified RSK correspondence for multisegments. Let $n \geq 0$ be an integer and $\lambda=\left(\lambda_{1}, \ldots, \lambda_{k}\right)$ a partition of $n$, i.e., $\lambda_{1} \geq \cdots \geq \lambda_{k}>0$ are integers and $\lambda_{1}+\cdots+\lambda_{k}=n$. The partition $\lambda$ gives rise to a Young diagram of size $n$. The conjugate partition $\lambda^{t}:=\mu=\left(\mu_{1}, \ldots, \mu_{l}\right)$ is given by $l=\lambda_{1}, \mu_{j}=\max \left\{i: \lambda_{i} \geq j\right\}$.

A semistandard Young tableau of shape $\lambda$ is a filling of a Young diagram of shape $\lambda$ by integers, such that the entries along each row are weakly increasing and the entries down each column are strictly increasing.

The classical Robinson-Schensted-Knuth (RSK) correspondence is a bijection between $\mathbb{N}(\mathbb{Z} \times \mathbb{Z})$ (i.e., multisets of pairs of integers) and pairs of semistandard Young tableaux of the same shape. We refer to [8, §4] or [27, §7.11-13] for standard references on RSK.

We will consider a slight modification of RSK where semistandard Young tableaux are replaced by inverted Young tableaux. By definition, an inverted Young tableau of shape $\lambda$ is a filling of the Young diagram of $\lambda$ by integers such that the entries along each row are strictly decreasing and the entries down each column are weakly decreasing. In other words, it is a double sequence $z_{i, j} \in \mathbb{Z}, i=1, \ldots, k$, $j=1, \ldots, \lambda_{i}$, that satisfies

$$
\begin{aligned}
& z_{i, 1}>\cdots>z_{i, \lambda_{i}}, \quad \forall i=1, \ldots, k \\
& z_{1, j} \geq \cdots \geq z_{\mu_{j}, j}, \quad \forall j=1, \ldots, l .
\end{aligned}
$$


We denote by $\mathcal{T}$ the set of pairs of inverted Young tableaux $(P, Q)$ of the same shape. (See [23] for a similar nonstandard convention. The appendix of [8] also discusses closely related variants of RSK.)

Thus, the modified RSK correspondence is a bijection

$$
\mathrm{RSK}^{\prime}: \mathbb{N}(\mathbb{Z} \times \mathbb{Z}) \longrightarrow \mathcal{T}
$$

It can be defined using a modification of the Schensted insertion/bumping algorithm where we replace strict inequalities by weak inequalities in the opposite direction and vice versa. It is advantageous, however, to use a modification of the Knuth algorithm which we will recall below. We remark that if $\operatorname{RSK}^{\prime}\left(\sum_{i \in I}\left(a_{i}, b_{i}\right)\right)=$ $(P, Q)$, then the $a_{i}$ 's and the $b_{i}$ 's comprise the entries of the tableaux $P$ and $Q$, respectively.

We may identify Seg as a subset of $\mathbb{Z} \times \mathbb{Z}$ by $\Delta \mapsto(b(\Delta), e(\Delta))$. Hence, we may identify $\mathfrak{M}$ with a subset of $\mathbb{N}(\mathbb{Z} \times \mathbb{Z})$. Thus, for any multisegment $\mathfrak{m} \in \mathfrak{M}$ we may consider the pair of inverted Young tableaux

$$
\left(P_{\mathfrak{m}}, Q_{\mathfrak{m}}\right):=\operatorname{RSK}^{\prime}(\mathfrak{m}) \in \mathcal{T} \text {. }
$$

In what follows we will only consider the restriction of $\mathrm{RSK}^{\prime}$ to $\mathfrak{M}$.

2.2.1. Ladders and tableaux. First, we would like to be able to describe certain elements of $\mathcal{T}$ in terms of ladders.

Let $\mathfrak{l}_{1}=\sum_{i=0}^{k} \Delta_{i}$ and $\mathfrak{l}_{2}=\sum_{i=0}^{k^{\prime}} \Delta_{i}^{\prime}$ be two ladders in standard form. We say that $\mathfrak{l}_{1}$ dominates $\mathfrak{l}_{2}$, if $k \geq k^{\prime}$ and $\overleftarrow{\Delta}_{i}^{\prime} \ll \Delta_{i}$ for all $i=0, \ldots, k^{\prime}$. We say that the pair $\left(\mathfrak{l}_{1}, \mathfrak{l}_{2}\right)$ is permissible if $\mathfrak{l}_{1}$ dominates $\mathfrak{l}_{2}$ and for all $i=0, \ldots, k^{\prime}$ and $j=0, \ldots, k$ such that $\overleftarrow{\Delta}_{i}^{\prime} \ll \Delta_{j}$ and $j$ maximal (i.e., either $j=k$ or $\overleftarrow{\Delta}_{i}^{\prime} \ll \Delta_{j+1}$; in particular, $j \geq i)$, we have $e\left(\Delta_{r}^{\prime}\right) \geq b\left(\Delta_{j-i+r}\right)$ for all $r=0, \ldots, i$.

Let us write $\mathcal{L}^{\prime}$ for the collection of tuples $\left(\mathfrak{l}_{1}, \ldots, \mathfrak{l}_{m}\right) \in \operatorname{Lad}^{m}$, for some $m$, such that $\mathfrak{l}_{i}$ dominates $\mathfrak{l}_{i+1}$, for all $1 \leq i<m$.

We may think of $\mathcal{L}^{\prime}$ as a subset of $\mathcal{T}$ as follows. Given $\underline{\mathfrak{l}}=\left(\mathfrak{l}_{1}, \ldots, \mathfrak{l}_{m}\right) \in \mathcal{L}^{\prime}$ where $\mathfrak{l}_{i}=\sum_{j=1}^{n_{i}} \Delta_{j}^{i}$ with $\Delta_{j+1}^{i} \ll \Delta_{j}^{i}$ we construct

$$
(P(\underline{l}), Q(\underline{\mathfrak{l}})) \in \mathcal{T}
$$

by letting the $(i, j)$-th entry in $P(\underline{\mathfrak{l}})$ (resp. $Q(\underline{\mathfrak{l}}))$ be $b\left(\Delta_{j}^{i}\right)$ (resp. $e\left(\Delta_{j}^{i}\right)$ ). The map $\underline{\mathfrak{l}} \mapsto(P(\mathfrak{l}), Q(\mathfrak{l}))$ is an injection of $\mathcal{L}^{\prime}$ into $\mathcal{T}$. Its image is the set of pairs $(P, Q)$ of tableaux of the same shape such that $P_{i, j} \leq Q_{i, j}$ for all entries of the tableaux.

Finally, we denote by $\mathcal{L} \subseteq \mathcal{L}^{\prime}$ the subset consisting of tuples $\left(\mathfrak{l}_{1}, \ldots, \mathfrak{l}_{m}\right) \in \mathcal{L}^{\prime}$ such that $\left(\mathfrak{l}_{i}, \mathfrak{l}_{j}\right)$ is permissible for all $1 \leq i<j \leq m$.

2.2.2. The Knuth implementation. Let us fix a multisegment $0 \neq \mathfrak{m}=\sum_{i \in I} \Delta_{i} \in$ $\mathfrak{M}$. We will explicate $\left(P_{\mathfrak{m}}, Q_{\mathfrak{m}}\right)$ introducing some terminology for multisegments.

For any $i \in I$ we define its depth to be the maximal number of segments in a ladder sub-multisegment of $\mathfrak{m}$ ending with $\Delta_{i}$, minus one. In other words, the function

is given by

$$
\mathfrak{d}=\mathfrak{d}_{\mathfrak{m}}: I \rightarrow \mathbb{Z}_{\geq 0}
$$

$$
\mathfrak{d}(i)=\max \left\{j: \exists i_{0}=i, i_{1}, \ldots, i_{j} \in I \text { such that } \Delta_{i_{r}} \ll \Delta_{i_{r+1}}, r=0, \ldots, j-1\right\} .
$$

We let $d=d(\mathfrak{m})=\max _{i \in I} \mathfrak{d}(i)$ be the depth of $\mathfrak{m}$.

Note that if $\mathfrak{l}=\sum_{i=0}^{n} \Delta_{i}$ is a ladder in standard form, then $\mathfrak{d}_{\mathfrak{l}}(i)=i$ for all $i$. 
In general, it is clear that if $\mathfrak{d}(i)=\mathfrak{d}(j)$, then either $\Delta_{i}=\Delta_{j}$ or $\Delta_{i} \supsetneq \Delta_{j}$ or $\Delta_{i} \subsetneq \Delta_{j}$.

We record some simple properties of the depth function in the following

\section{Lemma 2.1.}

(1) For any $i \in I$ and $0 \leq k<\mathfrak{d}(i)$ there exists $i^{\prime} \in I$ such that $\Delta_{i} \ll \Delta_{i^{\prime}}$ and $\mathfrak{d}\left(i^{\prime}\right)=k$.

(2) Suppose that $b\left(\Delta_{i}\right)<b\left(\Delta_{j}\right)$ and $\mathfrak{d}(i) \leq \mathfrak{d}(j)$ for some $i, j \in I$. Then, $\Delta_{j} \subsetneq \Delta_{i}$.

(3) Suppose that $b\left(\Delta_{i}\right) \leq b\left(\Delta_{j}\right)$ and $\mathfrak{d}(i)<\mathfrak{d}(j)$ for some $i, j \in I$. Then, $\Delta_{j} \subsetneq \Delta_{i}$.

(4) Suppose that $e\left(\Delta_{i}\right)<e\left(\Delta_{j}\right)$ and $\mathfrak{d}(i) \leq \mathfrak{d}(j)$ for some $i, j \in I$. Then, $\Delta_{i} \subsetneq \Delta_{j}$.

(5) Suppose that $e\left(\Delta_{i}\right) \leq e\left(\Delta_{j}\right)$ and $\mathfrak{d}(i)<\mathfrak{d}(j)$ for some $i, j \in I$. Then, $\Delta_{i} \subsetneq \Delta_{j}$.

(6) Let $i_{1}, i_{2} \in I$ be such that $\mathfrak{d}\left(i_{1}\right)=\mathfrak{d}\left(i_{2}\right)$ and $\Delta_{i_{2}} \subseteq \Delta_{i_{1}}$. Then, for any $i \in I$ such that $\Delta_{i_{2}} \subsetneq \Delta_{i} \subsetneq \Delta_{i_{1}}$ and $\mathfrak{d}(i) \geq \mathfrak{d}\left(i_{1}\right)$, there exists $j \in I$ such that $\Delta_{i_{2}} \subsetneq \Delta_{j} \subsetneq \Delta_{i_{1}}$ and $\mathfrak{d}(j)=\mathfrak{d}\left(i_{1}\right)$.

Proof. The first part is clear.

Suppose that $b\left(\Delta_{i}\right)<b\left(\Delta_{j}\right)$. Then either $\Delta_{i} \ll \Delta_{j}$, in which case $\mathfrak{d}(i)>\mathfrak{d}(j)$, or $e\left(\Delta_{i}\right) \geq e\left(\Delta_{j}\right)$ in which case $\Delta_{j} \subsetneq \Delta_{i}$. This proved part (2)

Part (3) follows from part (2) and the fact that if $b\left(\Delta_{i}\right)=b\left(\Delta_{j}\right)$ and $e\left(\Delta_{i}\right)<$ $e\left(\Delta_{j}\right)$ then $\mathfrak{d}(j) \leq \mathfrak{d}(i)$.

Parts (4) and (5) are proved analogously.

The last part is clear if $\mathfrak{d}(i)=\mathfrak{d}\left(i_{1}\right)$. Otherwise, by the first part, there exists $j \in I$ such that $\Delta_{i} \ll \Delta_{j}$ and $\mathfrak{d}(j)=\mathfrak{d}\left(i_{1}\right)$. Then, $b\left(\Delta_{i_{1}}\right) \leq b\left(\Delta_{i}\right)<b\left(\Delta_{j}\right)$ and $e\left(\Delta_{i_{2}}\right) \leq e\left(\Delta_{i}\right)<e\left(\Delta_{j}\right)$. By parts (2) and (4) we have $\Delta_{i_{2}} \subsetneq \Delta_{j} \subsetneq \Delta_{i_{1}}$, as required.

For any $k=0, \ldots, d$, choose an admissible enumeration $\left\{i_{1}^{k}, \ldots, i_{l}^{k}\right\}$ of $\mathfrak{d}^{-1}(k)$, namely such that $\Delta_{i_{1}^{k}} \supseteq \ldots \supseteq \Delta_{i_{l}^{k}}$, and set $j_{k}:=i_{l}^{k}$. (Of course, $l$ depends on $k$.)

Denote by $i \mapsto i^{\vee}$ the permutation of the index set $I$, whose cycle decomposition is given by $\left\{\left(i_{1}^{k}, \ldots, i_{l}^{k}\right)\right\}_{k=0, \ldots, d}$.

Define

$$
\begin{gathered}
\Delta_{i}^{\prime}=\left[b\left(\Delta_{i}\right), e\left(\Delta_{i^{\vee}}\right)\right] \in \text { Seg }, \quad i \in I, \\
I^{\natural}=\left\{j_{0}, \ldots, j_{d}\right\}, I^{\prime}=I \backslash I^{\natural}, \\
\mathfrak{l}(\mathfrak{m})=\sum_{i \in I^{\natural}} \Delta_{i}^{\prime}, \mathfrak{m}^{\prime}=\sum_{i \in I^{\prime}} \Delta_{i}^{\prime} \in \mathfrak{M} .
\end{gathered}
$$

Note that for any $i \in I^{\natural}$ we have

$$
b\left(\Delta_{i}^{\prime}\right)=\max _{j \in I: \mathfrak{d}(j)=\mathfrak{d}(i)} b\left(\Delta_{j}\right), \quad e\left(\Delta_{i}^{\prime}\right)=\max _{j \in I: \mathfrak{d}(j)=\mathfrak{d}(i)} e\left(\Delta_{j}\right) .
$$

Thus, it follows from Lemma 2.1(1) that $\mathfrak{l}(\mathfrak{m})$ is a ladder.

Also, with the notation above,

$$
\Delta_{i_{1}^{k}}^{\prime} \supseteq \ldots \supseteq \Delta_{i_{l-1}^{k}}^{\prime}
$$

and

$$
e\left(\Delta_{i}^{\prime}\right) \leq e\left(\Delta_{i}\right) \quad \forall i \in I^{\prime}
$$




\section{Lemma 2.2.}

(1) Suppose that $i, j \in I$ are such that $\Delta_{i \vee} \subsetneq \Delta_{j} \subsetneq \Delta_{i}$. Then, $\mathfrak{d}(j)<\mathfrak{d}(i)$. In particular, $b\left(\Delta_{j}\right)>b\left(\Delta_{i}\right)$.

(2) Suppose that $b\left(\Delta_{j}\right)>b\left(\Delta_{i}\right)$ and $e\left(\Delta_{i}^{\prime}\right)<e\left(\Delta_{j}\right)$ for some indices $i, j \in I$. Then $\mathfrak{d}(j)<\mathfrak{d}(i)$.

(3) Suppose that $b\left(\Delta_{j}\right) \geq b\left(\Delta_{i}\right)$ and $e\left(\Delta_{i}^{\prime}\right) \leq e\left(\Delta_{j}\right)$ for some indices $i, j \in I$. Then $\mathfrak{d}(j) \leq \mathfrak{d}(i)$.

Proof. (1) Assume on the contrary that $\mathfrak{d}(j) \geq \mathfrak{d}(i)$. By Lemma 2.1](6), we may assume without loss of generality that $\mathfrak{d}(j)=\mathfrak{d}(i)$. This contradicts the definition of $i^{\vee}$.

The last statement follows from part (3) of Lemma 2.1

(2) Assume on the contrary that $\mathfrak{d}(j) \geq \mathfrak{d}(i)$. By parts (2) and (4) of Lemma 2.1 we have $\Delta_{i \vee} \subsetneq \Delta_{j} \subsetneq \Delta_{i}$, in contradiction with the previous part.

(3) The last part is proved similarly using Lemma 2.1, parts (3) and (5).

Corollary 2.3. For all $i \in I^{\prime}$ we have $\mathfrak{d}_{\mathfrak{m}^{\prime}}(i) \leq \mathfrak{d}_{\mathfrak{m}}(i)$. Moreover, if $\Delta_{i}^{\prime} \ll \Delta_{j}^{\prime}$ with $i, j \in I^{\prime}$, then $\mathfrak{d}_{\mathfrak{m}}(i)>\mathfrak{d}_{\mathfrak{m}}(j)$.

Proof. Clearly, the first part follows from the second part. If $\Delta_{i}^{\prime} \ll \Delta_{j}^{\prime}$, then $b\left(\Delta_{j}\right)=b\left(\Delta_{j}^{\prime}\right)>b\left(\Delta_{i}^{\prime}\right)=b\left(\Delta_{i}\right)$ and $e\left(\Delta_{i}^{\prime}\right)<e\left(\Delta_{j}^{\prime}\right) \leq e\left(\Delta_{j}\right)$ by (2.4). It follows from Lemma 2.2(2) that $\mathfrak{d}_{\mathfrak{m}}(i)>\mathfrak{d}_{\mathfrak{m}}(j)$.

We define a map

$$
\mathcal{K}: \mathfrak{M} \backslash\{0\} \rightarrow \operatorname{Lad} \times \mathfrak{M}, \quad \mathcal{K}(\mathfrak{m})=\left(\mathfrak{l}(\mathfrak{m}), \mathfrak{m}^{\prime}\right) .
$$

It is clear that $\mathcal{K}(\mathfrak{m})$ is well defined (i.e., does not depend on the choice of admissible enumerations of the fibers of $\mathfrak{d})$. We call $\mathfrak{l}(\mathfrak{m})$ the highest ladder of $\mathfrak{m}$, and $\mathfrak{m}^{\prime}$ the derived multisegment of $\mathfrak{m}$.

Define recursively

$$
\mathcal{R S K}: \mathfrak{M} \rightarrow \mathcal{L}^{\prime}
$$

by

$$
\mathcal{R} \mathcal{S K}(0)=\emptyset, \quad \mathcal{R} \mathcal{S} \mathcal{K}(\mathfrak{m})=\left(\mathfrak{l}(\mathfrak{m}), \mathcal{R} \mathcal{S} \mathcal{K}\left(\mathfrak{m}^{\prime}\right)\right), \quad \mathfrak{m} \neq 0 .
$$

This is well defined since $\left|\mathfrak{m}^{\prime}\right|<|\mathfrak{m}|$. We shall see momentarily that indeed $\mathcal{R} \mathcal{S K}(\mathfrak{m}) \in \mathcal{L}^{\prime}$ (and in fact $\left.\mathcal{R} \mathcal{S K}(\mathfrak{m}) \in \mathcal{L}\right)$ - see Proposition 2.4.

Adapting the discussion of [13, §4] to our conventions we obtain that

$$
\operatorname{RSK}^{\prime}(\mathfrak{m})=(P(\mathcal{R S K}(\mathfrak{m})), Q(\mathcal{R S K}(\mathfrak{m}))) .
$$

2.2.3. On the image of $\mathcal{R} \mathcal{S} \mathcal{K}$. The fact that we consider only multisegments rather than multisets of arbitrary pairs of integers means that there are restrictions on the image of $\mathcal{R S K}$. This situation is explained in Appendix A where a full characterization of the image of (a certain variant of) $\mathcal{R S K}$ is given by means of key tableaux in the sense of Lascoux and Schützenberger, a notion which has links to the theory of Demazure crystals.

A partial characterization from a somewhat different point of view will be given here, and will be relevant for the core of our arguments. A combined understanding of both these approaches may be beneficial for a better understanding of the entire theme of this paper. We leave it for future endeavors. 
Let $\mathfrak{m}$ be a multisegment and $\mathfrak{l}$ a ladder. We say that $\mathfrak{l}$ dominates $\mathfrak{m}$, if $\mathfrak{l}$ dominates any ladder sub-multisegment of $\mathfrak{m}$. It is clear that this terminology is consistent with the previous one in the case where $\mathfrak{m}$ itself is a ladder. Writing $\mathfrak{l}=\sum_{j=0}^{n} \Delta_{j}$ in standard form and $\mathfrak{m}=\sum_{i \in I} \Delta_{i}$ where $I \cap\{0, \ldots, n\}=\emptyset$, it follows from the definition of depth that

$$
\mathfrak{l} \text { dominates } \mathfrak{m} \Longleftrightarrow \overleftarrow{\Delta}_{i} \ll \Delta_{\mathfrak{d}_{\mathfrak{m}}(i)} \text { for all } i \in I
$$

We say that the pair $(\mathfrak{l}, \mathfrak{m})$ is permissible if $\left(\mathfrak{l}, \mathfrak{l}^{\prime}\right)$ is permissible (and in particular, $\mathfrak{l}$ dominates $\mathfrak{l}^{\prime}$ ) for any ladder sub-multisegment $\mathfrak{l}^{\prime}$ of $\mathfrak{m}$. Once again, this is consistent with the previous terminology if $\mathfrak{m}$ is itself a ladder. Writing $\mathfrak{l}$ and $\mathfrak{m}$ as above, it is easy to see that $(\mathfrak{l}, \mathfrak{m})$ is permissible if and only if $\mathfrak{l}$ dominates $\mathfrak{m}$ and for every ladder sub-multisegment $\Delta_{i_{k}} \ll \Delta_{i_{k-1}} \ll \ldots \ll \Delta_{i_{0}}, i_{0}, \ldots, i_{k} \in I$ of $\mathfrak{m}$ there exists $j \leq n-k$ such that $e\left(\Delta_{i_{0}}\right) \geq b\left(\Delta_{j}\right)$ and $\overleftarrow{\Delta}_{i_{k}} \ll \Delta_{j+k}$

Denote by $\mathfrak{A} \subseteq$ Lad $\times \mathfrak{M}$ the set of permissible pairs.

Proposition 2.4. The map $\mathcal{K}$ defines a bijection

$$
\mathcal{K}: \mathfrak{M} \backslash\{0\} \rightarrow \mathfrak{A}
$$

Moreover, the map $\mathcal{R S K}$ is injective and its image is contained in $\mathcal{L}$.

Remark 2.5. The map $\mathcal{R} \mathcal{S} \mathcal{K}$ is not onto $\mathcal{L}$. For instance, $([3,3]+[1,2],[2,3],[1,2])$ is not in the image of $\mathcal{R S K}$. Indeed, otherwise there would exist $\mathfrak{m}$ such that $\mathcal{K}(\mathfrak{m})=\left([3,3]+[1,2], \mathfrak{m}^{\prime}\right) \in \mathfrak{A}$ and $\mathcal{R} \mathcal{S} \mathcal{K}\left(\mathfrak{m}^{\prime}\right)=([2,3],[1,2])$. Since $\mathcal{R S} \mathcal{K}([1,3]+$ $[2,2])=([2,3],[1,2])$ and $\mathcal{R} \mathcal{S} \mathcal{K}$ is injective, this means that $\mathfrak{m}^{\prime}=[1,3]+[2,2]$. However, $([3,3]+[1,2],[1,3]+[2,2])$ is not permissible and we get a contradiction.

On the other hand, if we restrict $\mathcal{R} \mathcal{S} \mathcal{K}$ to the set of multisegments $\mathfrak{m}=\sum_{i \in I} \Delta_{i}$ such that $b\left(\Delta_{i}\right) \leq e\left(\Delta_{j}\right)$ for all $i, j \in I$, then the image consists of the subset of $\mathcal{L}^{\prime}$ consisting of the tuples $\left(\mathfrak{l}_{1}, \ldots, \mathfrak{l}_{m}\right) \in \mathcal{L}^{\prime}$ such that $b(\Delta) \leq e\left(\Delta^{\prime}\right)$ for any $\Delta$ and $\Delta^{\prime}$ which occur in $\sum \mathfrak{l}_{i}$. We refer to this as the saturated case.

Proof. Fix $0 \neq \mathfrak{m} \in \mathfrak{M}$ and use the notation as before. We write $\mathfrak{d}=\mathfrak{d}_{\mathfrak{m}}$. We also write $I^{\natural}=\left\{j_{0}, \ldots, j_{d}\right\}$ where $d=d(\mathfrak{m})$ and $\mathfrak{d}\left(j_{r}\right)=r$ for all $r$.

We first show that $\mathfrak{l}(\mathfrak{m})$ dominates $\mathfrak{m}^{\prime}$. Let $j \in I^{\prime}$ and $r=\mathfrak{d}(j)$. By (2.2) we have $\overleftarrow{\Delta}_{j} \ll \Delta_{j_{r}}^{\prime}$. Since $b\left(\Delta_{j}^{\prime}\right)=b\left(\Delta_{j}\right)$ and $e\left(\Delta_{j}^{\prime}\right) \leq e\left(\Delta_{j}\right)$ it follows that

$$
\overleftarrow{\Delta}_{j}^{\prime} \ll \Delta_{j_{r}}^{\prime}
$$

It now follows from Corollary 2.3 and (2.5) that $\mathfrak{l}(\mathfrak{m})$ dominates $\mathfrak{m}^{\prime}$.

Note that $e\left(\Delta_{j}\right) \geq b\left(\Delta_{i}\right)$ for all $i, j \in I$ with $\mathfrak{d}(i)=\mathfrak{d}(j)$. Hence, by (2.2) and the fact that $\mathfrak{l}(\mathfrak{m})$ is a ladder, we see that $e\left(\Delta_{j}^{\prime}\right) \geq b\left(\Delta_{j_{r}}^{\prime}\right)$ for any $j \in I^{\prime}$ such that $\mathfrak{d}(j) \leq r$.

To show that $\left(\mathfrak{l}(\mathfrak{m}), \mathfrak{m}^{\prime}\right)$ is permissible, suppose that $\Delta_{i_{k}}^{\prime} \ll \Delta_{i_{k-1}}^{\prime} \ll \ldots \ll \Delta_{i_{0}}^{\prime}$ with $i_{0}, \ldots, i_{k} \in I^{\prime}$. Let $r=\mathfrak{d}\left(i_{k}\right)$. By (2.6) we have $\overleftarrow{\Delta}_{i_{k}}^{\prime} \ll \Delta_{j_{r}}^{\prime}$. On the other hand, by Corollary 2.3 we have $r \geq \mathfrak{d}\left(i_{0}\right)+k$. It follows from the above that $e\left(\Delta_{i_{0}}^{\prime}\right) \geq b\left(\Delta_{j_{r-k}}^{\prime}\right)$. Hence, $\left(\mathfrak{l}(\mathfrak{m}), \mathfrak{m}^{\prime}\right)$ is permissible.

In conclusion, $\mathcal{K}(\mathfrak{m}) \in \mathfrak{A}$.

In order to show that $\mathcal{K}$ is a bijection, we describe the inverse $\mathcal{K}^{\prime}: \mathfrak{A} \rightarrow \mathfrak{M} \backslash\{0\}$ following [8, §4.2]. 
Let $(\mathfrak{l}, \mathfrak{m}) \in \mathfrak{A}$. Write $\mathfrak{l}=\sum_{j=0}^{n} \Delta_{j}$ in standard form and $\mathfrak{m}=\sum_{i \in I} \Delta_{i}$ with $I \cap J=\emptyset$ where $J=\{0, \ldots, n\}$. We define $g=g_{\mathfrak{m}, \mathfrak{l}}: I \rightarrow J$ by

$$
g(i)=\max \left\{j \in J: \overleftarrow{\Delta}_{i} \ll \Delta_{j}\right\}
$$

Since $\mathfrak{l}$ dominates $\mathfrak{m}, g$ is well-defined and $g(i) \geq \mathfrak{d}(i)$ for all $i$ by (2.5). Now define $f=f_{\mathfrak{m}, \mathfrak{l}}: I \rightarrow J$ by

$f(i)=\min \left\{g\left(i_{k}\right)-k: \exists i=i_{0}, \ldots, i_{k} \in I\right.$ such that $\left.\Delta_{i_{r+1}} \ll \Delta_{i_{r}}, r=0, \ldots, k-1\right\}$.

Thus,

$$
f(i)=\min \left(g(i),\left\{f(j)-1: j \in I, \Delta_{j} \ll \Delta_{i}\right\}\right) .
$$

Once again, it follows from (2.5) that $f(i) \geq \mathfrak{d}(i)$ for all $i$. Moreover, for any $j \in J$ we may write the fiber $Y_{j}=f^{-1}(j)$ as $Y_{j}=\left\{i_{1}, \ldots, i_{k}\right\}$ (possibly with $k=0$ ) where $\Delta_{i_{r+1}} \subseteq \Delta_{i_{r}}, r=1, \ldots, k-1$. For all $r=1, \ldots, k$ we have $\overleftarrow{\Delta}_{i_{r}} \ll \Delta_{j}$ (since $\left.j \leq g\left(i_{r}\right)\right)$ and moreover, $e\left(\Delta_{i_{r}}\right) \geq b\left(\Delta_{j}\right)$ by the permissibility of $(\mathfrak{l}, \mathfrak{m})$.

Let $\sigma$ be the permutation of $I \cup J$ whose cycles are $\left(i_{1}, \ldots, i_{k}, j\right)$ as we vary over $j \in J$.

For any $i \in I \cup J$, we set $\Delta_{i}^{\boldsymbol{中}}=\left[b\left(\Delta_{\sigma(i)}\right), e\left(\Delta_{i}\right)\right]$. Note that we still have $\Delta_{i_{r+1}}^{\mathbf{q}} \subseteq \Delta_{i_{r}}^{\mathbf{k}}, r=1, \ldots, k-1 ;$ also $\Delta_{i_{1}}^{\mathbf{k}} \subseteq \Delta_{j}^{\mathbf{q}}$.

Finally, set $\mathcal{K}^{\prime}(\mathfrak{l}, \mathfrak{m})=\sum_{i \in I \cup J} \Delta_{i}^{\mathfrak{q}}$. It is easy to see that $\mathcal{K}^{\prime}$ is the inverse of $\mathcal{K}$.

The injectivity of $\mathcal{K}$ implies the injectivity of $\mathcal{R} \mathcal{S K}$ by virtue of its recursive definition. In order to prove the last statement of the proposition, we need to show (again, by the recursive nature of $\mathcal{R S} \mathcal{S}$ ) that for any pair $(\mathfrak{l}, \mathfrak{m}) \in \mathfrak{A}$ with $\mathfrak{m} \neq 0$, both $(\mathfrak{l}, \mathfrak{l}(\mathfrak{m}))$ and $\left(\mathfrak{l}, \mathfrak{m}^{\prime}\right)$ are in $\mathfrak{A}$ as well.

Suppose that $(\mathfrak{l}, \mathfrak{m}) \in \mathfrak{A}$. It easily follows from (2.5) and (2.2) that $\mathfrak{l}$ dominates $\mathfrak{l}(\mathfrak{m})$.

Write $\mathfrak{l}$ and $\mathfrak{m}$ as above and fix $s \leq d(\mathfrak{m})$. Let $i=0, \ldots, n$ be the maximal index such that $\overleftarrow{\Delta}_{j_{s}}^{\prime} \ll \Delta_{i}$. By (2.2), $\overleftarrow{\Delta}_{j_{s}} \ll \Delta_{i}$ and $\overleftarrow{\Delta}_{j_{s}^{\vee}} \ll \Delta_{i}$. Suppose that $i<n$. Since $\overleftarrow{\Delta}_{j_{s}}^{\prime} \ll \Delta_{i+1}$ we cannot have both $\overleftarrow{\Delta}_{j_{s}} \ll \Delta_{i+1}$ and $\overleftarrow{\Delta}_{j_{s}^{\vee}} \ll \Delta_{i+1}$. Let $j^{\prime} \in\left\{j_{s}, j_{s}^{\vee}\right\}$ be such that $\overleftarrow{\Delta}_{j^{\prime}} \nless \Delta_{i+1}$. If $i=n$, then we take either $j^{\prime}=j_{s}$ or $j_{s}^{\vee}$ - it makes no difference. In either case, there exist indices $k_{0}, \ldots, k_{s}=j^{\prime}$ in $I$ such that $\Delta_{k_{r}} \ll \Delta_{k_{r-1}}, r=1, \ldots, s$ and $\mathfrak{d}\left(\Delta_{k_{r}}\right)=r$ for all $r$. Since $(\mathfrak{l}, \mathfrak{m})$ is permissible, for any $r \leq s$ we have $e\left(\Delta_{k_{r}}\right) \geq b\left(\Delta_{i-s+r}\right)$. This implies that $e\left(\Delta_{j_{r}}^{\prime}\right) \geq b\left(\Delta_{i-s+r}\right)$ by (2.2). It follows that $(\mathfrak{l}, \mathfrak{l}(\mathfrak{m}))$ is permissible.

Let $i \in I^{\prime}$. Then, by (2.5) and Corollary 2.3.

$$
b\left(\Delta_{i}^{\prime}\right)=b\left(\Delta_{i}\right) \leq b\left(\Delta_{\mathfrak{d}(i)}\right) \leq b\left(\Delta_{\mathfrak{o}_{\mathfrak{m}^{\prime}}(i)}\right)
$$

and

$$
e\left(\Delta_{i}^{\prime}\right)=e\left(\Delta_{i \vee}\right) \leq e\left(\Delta_{\mathfrak{d}\left(i^{\vee}\right)}\right)=e\left(\Delta_{\mathfrak{d}(i)}\right) \leq e\left(\Delta_{\mathfrak{d}_{\mathfrak{m}^{\prime}}(i)}\right) .
$$

It follows (again by (2.5) ) that $\mathfrak{l}$ dominates $\mathfrak{m}^{\prime}$.

Next, suppose that

$$
\Delta_{i_{k}}^{\prime} \ll \Delta_{i_{k-1}}^{\prime} \ll \ldots \ll \Delta_{i_{0}}^{\prime}
$$

for some indices $i_{0}, \ldots, i_{k} \in I^{\prime}$. We will construct a ladder sub-multisegment

$$
\Delta_{s_{k}} \ll \Delta_{s_{k-1}} \ll \ldots \ll \Delta_{s_{0}}
$$

of $\mathfrak{m}$ with $s_{k}=i_{k}^{\vee}$ such that $\mathfrak{d}\left(s_{r}\right)=\mathfrak{d}\left(i_{r}\right)$ and $e\left(\Delta_{s_{r}}\right) \leq e\left(\Delta_{i_{r}}^{\prime}\right)$ for all $r$. 
We define $s_{r}, r<k$ by descending induction on $r$. Given $s_{r} \in I$ with $r>0$, let $j=i_{r-1}^{\vee}$, so that $\mathfrak{d}(j)=\mathfrak{d}\left(i_{r-1}\right)$ and $e\left(\Delta_{j}\right)=e\left(\Delta_{i_{r-1}}^{\prime}\right)<e\left(\Delta_{i_{r}}^{\prime}\right) \geq e\left(\Delta_{s_{r}}\right)$. By Corollary 2.3 $\mathfrak{d}(j)=\mathfrak{d}\left(i_{r-1}\right)>\mathfrak{d}\left(i_{r}\right)=\mathfrak{d}\left(s_{r}\right)$. By Lemma 2.1(1), there exists $s_{r-1} \in I$ such that $\mathfrak{d}\left(s_{r-1}\right)=\mathfrak{d}(j)$ and $\Delta_{s_{r}} \ll \Delta_{s_{r-1}}$. If $\Delta_{s_{r}} \ll \Delta_{j}$, take $s_{r-1}=j$. Otherwise, $b\left(\Delta_{j}\right) \leq b\left(\Delta_{s_{r}}\right)<b\left(\Delta_{s_{r-1}}\right)$ and therefore, by Lemma 2.1(2) $\Delta_{s_{r-1}} \subsetneq$ $\Delta_{j}$. In any case, $e\left(\Delta_{j}\right) \geq e\left(\Delta_{s_{r-1}}\right)$ as required.

By the permissibility of $(\mathfrak{l}, \mathfrak{m})$, there exists $j \in\{0, \ldots, n\}$ such that $\overleftarrow{\Delta}_{s_{k}} \ll \Delta_{j}$ and $e\left(\Delta_{s_{0}}\right) \geq b\left(\Delta_{j-k}\right)$. Since $e\left(\Delta_{i_{k}}^{\prime}\right)=e\left(\Delta_{s_{k}}\right)$ and $b\left(\Delta_{i_{k}}^{\prime}\right) \leq b\left(\Delta_{s_{k}}\right)$, we have $\overleftarrow{\Delta}_{i_{k}}^{\prime} \ll$ $\Delta_{j}$. Also, $e\left(\Delta_{i_{0}}^{\prime}\right) \geq e\left(\Delta_{s_{0}}\right) \geq b\left(\Delta_{j-k}\right)$. It follows that $\left(\mathfrak{l}, \mathfrak{m}^{\prime}\right)$ is permissible.

2.2.4. An inductive description. We finish our discussion on the RSK algorithm with Lemma 2.6, which allows for inductive arguments in certain cases.

Lemma 2.6. Assume that $\mathfrak{m} \neq 0$ and $\min \mathfrak{l}(\mathfrak{m})=\min \mathfrak{m}$. Let $d=d(\mathfrak{m})$ be the depth of $\mathfrak{m}$ and let $i_{0} \in \mathfrak{d}_{\mathfrak{m}}^{-1}(d)$ be such that $\Delta_{i_{0}} \supseteq \Delta_{i}$ for all $i \in \mathfrak{d}_{\mathfrak{m}}^{-1}(d)$. Then,

(1) $\mathfrak{d}_{\mathfrak{m}}^{-1}(d)=\left\{i \in I: b\left(\Delta_{i}\right)=\min \mathfrak{m}, \Delta_{i_{0}} \supseteq \Delta_{i}\right\}$. In particular, $b\left(\Delta_{i_{0}}\right)=$ $\min \mathfrak{m}$.

(2) $\Delta_{i_{0}} \leq \mathfrak{l}(\mathfrak{m})$.

(3)

$$
\sum_{i \in I^{\prime}: b\left(\Delta_{i}^{\prime}\right)=\min \mathfrak{m}, \Delta_{i}^{\prime} \subseteq \Delta_{i_{0}}} \Delta_{i}^{\prime}=\sum_{i \in \mathfrak{d}_{\mathfrak{m}}^{-1}(d) \backslash\left\{i_{0}\right\}} \Delta_{i} .
$$

(It is of course not excluded that $\mathfrak{d}_{\mathfrak{m}}^{-1}(d)=\left\{i_{0}\right\}$.)

$$
\mathcal{K}\left(\mathfrak{m}-\sum_{i \in \mathfrak{o}_{\mathfrak{m}}^{-1}(d)} \Delta_{i}\right)=\left(\mathfrak{l}(\mathfrak{m})-\Delta_{i_{0}}, \mathfrak{m}^{\prime}-\sum_{i \in \mathfrak{d}_{\mathfrak{m}}^{-1}(d) \backslash\left\{i_{0}\right\}} \Delta_{i}\right) .
$$

Proof. Let $\Delta$ be the segment in $\mathfrak{l}(\mathfrak{m})$ with $b(\Delta)=\min \mathfrak{l}(\mathfrak{m})=\min \mathfrak{m}$. By equation (2.2),$b\left(\Delta_{i}\right)=\min \mathfrak{m}$ for any $i \in \mathfrak{d}_{\mathfrak{m}}^{-1}(d)$. Similarly, $e(\Delta)=e\left(\Delta_{i_{0}}\right)$ and hence, $\Delta=\Delta_{i_{0}}$.

Part (1) now follows from the fact that $\mathfrak{d}_{\mathfrak{m}}(i) \geq \mathfrak{d}_{\mathfrak{m}}\left(i_{0}\right)$ for any $i \in I$ such that $b\left(\Delta_{i}\right)=\min \mathfrak{m}$ and $\Delta_{i_{0}} \supseteq \Delta_{i}$.

The rest of the lemma follows from the description of $\mathcal{K}(\mathfrak{m})$. For part (3) we also use the fact that if $i \in I^{\prime}, b\left(\Delta_{i}^{\prime}\right)=\min \mathfrak{m}$ and $\Delta_{i}^{\prime} \subseteq \Delta_{i_{0}}$, then $\mathfrak{d}_{\mathfrak{m}}(i)=d$. To show this, suppose on the contrary that $\mathfrak{d}_{\mathfrak{m}}(i)<d$. By part (1), $\Delta_{i_{0}} \subsetneq \Delta_{i}$. Also $\Delta_{i \vee} \subseteq \Delta_{i_{0}}$ and in fact $\Delta_{i \vee} \subsetneq \Delta_{i_{0}}$ since $b\left(\Delta_{i \vee}\right)>\min \mathfrak{m}$ (again, by part (10)). We get a contradiction to Lemma 2.2(1).

\section{Relation With the Meglin-Waldspurger involution}

3.1. Møglin-Waldspurger involution. Let us recall the Mœeglin-Waldspurger algorithm from [21, §II.2]. It will be more handy for us to consider the mirror image of the procedure of [loc. cit.], obtained simply by inverting all inequalities. This has no effect on the final result since the Møglin-Waldspurger involution is compatible with taking contragredient.

For any multisegment $0 \neq \mathfrak{m}=\sum_{i \in I} \Delta_{i} \in \mathfrak{M}$ we define a segment $\Delta^{\circ}(\mathfrak{m})$ and a multisegment $\mathfrak{m}^{\dagger}$ as follows.

Let $i_{1} \in I$ be such that

$$
b\left(\Delta_{i_{1}}\right)=\min \mathfrak{m} \text { and } e\left(\Delta_{i_{1}}\right) \text { is minimal with this property. }
$$


If there is no $i \in I$ such that $\Delta_{i_{1}} \ll \Delta_{i}$ and $b\left(\Delta_{i}\right)=b\left(\Delta_{i_{1}}\right)+1$, then we set $\Delta^{\circ}(\mathfrak{m}):=$ $[\min \mathfrak{m}, \min \mathfrak{m}] \in$ Seg. Otherwise, let $i_{2} \in I$ be such that $\Delta_{i_{1}} \ll \Delta_{i_{2}}, b\left(\Delta_{i_{2}}\right)=$ $b\left(\Delta_{i_{1}}\right)+1$ and $e\left(\Delta_{i_{2}}\right)$ is minimal with respect to these properties. Continuing this way, we define an integer $k>0$ and indices $i_{2}, \ldots, i_{k} \in I$, such that

For all $1 \leq j<k, \Delta_{i_{j}} \ll \Delta_{i_{j+1}}, b\left(\Delta_{i_{j+1}}\right)=b\left(\Delta_{i_{j}}\right)+1$ and $e\left(\Delta_{i_{j+1}}\right)$ is minimal with respect to these properties.

(3.1c) There does not exist $i \in I$ such that $\Delta_{i_{k}} \ll \Delta_{i}$ and $b\left(\Delta_{i}\right)=b\left(\Delta_{i_{k}}\right)+1$.

We set

$\Delta^{\circ}(\mathfrak{m}):=\left\{b\left(\Delta_{i_{1}}\right), \ldots, b\left(\Delta_{i_{k}}\right)\right\}=\left[b\left(\Delta_{i_{1}}\right), b\left(\Delta_{i_{k}}\right)\right]=[\min \mathfrak{m}, \min \mathfrak{m}+k-1] \in$ Seg.

We call $I^{*}=\left\{i_{1}, \ldots, i_{k}\right\} \subseteq I$ a set of leading indices of $\mathfrak{m}$. It is not unique, but the set $\left\{\Delta_{i_{1}}, \ldots, \Delta_{i_{k}}\right\}$ depends only on $\mathfrak{m}$. We also write $i_{\min }^{*}=i_{1}, i_{\max }^{*}=i_{k}$ and denote by $i \mapsto i^{+}: I^{*} \backslash\left\{i_{\max }^{*}\right\} \rightarrow I^{*} \backslash\left\{i_{\min }^{*}\right\}$ the bijection $i_{j} \mapsto i_{j+1}$. The inverse will be denoted by $i \mapsto i^{-}$.

We set $\mathfrak{m}^{\dagger}:=\sum_{i \in I} \Delta_{i}^{*} \in \mathfrak{M}$ (discarding the summands that are empty sets), where

$$
\Delta_{i}^{*}= \begin{cases}-\Delta_{i} & i \in I^{*}, \\ \Delta_{i} & \text { otherwise. }\end{cases}
$$

We denote the resulting map $\mathfrak{m} \mapsto\left(\mathfrak{m}^{\dagger}, \Delta^{\circ}(\mathfrak{m})\right)$ by

$$
\mathcal{M W}: \mathfrak{M} \backslash\{0\} \rightarrow \mathfrak{M} \times \mathrm{Seg} .
$$

We say that $\mathfrak{m}$ is non-degenerate if $\Delta_{i}^{*} \neq \emptyset$, for all $i \in I$. Equivalently, $\Delta_{i} \neq$ $[\min \mathfrak{m}, \min \mathfrak{m}]$ for all $i \in I$.

Applying the map $\mathcal{M W}$ repeatedly we obtain the Møglin-Waldspurger map $\mathfrak{m} \mapsto \mathfrak{m}^{\#}$ on $\mathfrak{M}$, which is the combinatorial counterpart of the Zelevinsky involution [33, §9]. More precisely, $\mathfrak{m}^{\#}$ is defined recursively by

$$
0^{\#}=0, \quad \mathfrak{m}^{\#}=\Delta^{\circ}(\mathfrak{m})+\left(\mathfrak{m}^{\dagger}\right)^{\#}, \mathfrak{m} \neq 0 .
$$

This is well-defined since $\left|\mathfrak{m}^{\dagger}\right|<|\mathfrak{m}|$. In fact, the map $\mathfrak{m} \mapsto \mathfrak{m} \#$ is an involution, although this is not obvious from the definition. In particular, the map $\mathcal{M W}$ is injective. (It is easy to write down explicitly the inverse of $\mathcal{M W}$ - see [17, Remark 3.14].)

The subset Lad of ladder multisegments is preserved under $\mathfrak{m} \mapsto \mathfrak{m} \#$.

3.2. We turn now to study the relations between the Mœeglin-Waldspurger algorithm and RSK.

Let $\mathfrak{m}=\sum_{i \in I} \Delta_{i} \in \mathfrak{M}$ be a nonzero multisegment. We will use the notation and terminology of the previous section, as well as that of $\$ 2.2 .2$.

We consider the more involved case that was not covered by Lemma 2.6, namely, when $\min \mathfrak{m}<\min l(\mathfrak{m})$. The key is the relation between the depth functions with respect to $\mathfrak{m}$ and $\mathfrak{m}^{\dagger}$, which is explicated as follows.

Lemma 3.1. Suppose that $\mathfrak{m}$ is non-degenerate and let $I^{*}$ be a set of leading indices for $\mathfrak{m}$. Then,

(1) For every $i \in I$ we have $\mathfrak{d}_{\mathfrak{m}}(i) \leq \mathfrak{d}_{\mathfrak{m}^{\dagger}}(i) \leq \mathfrak{d}_{\mathfrak{m}}(i)+1$. 
(2) Define

$I_{b}=\left\{i \in I: \exists i^{\prime} \in I^{*}\right.$ such that $b\left(\Delta_{i}\right)=b\left(\Delta_{i^{\prime}}\right), \Delta_{i} \subsetneq \Delta_{i^{\prime}}$ and $\left.\mathfrak{d}_{\mathfrak{m}}(i)=\mathfrak{d}_{\mathfrak{m}}\left(i^{\prime}\right)\right\}$.

Then, $I^{*}$ and $I_{b}$ are disjoint. Moreover, we have

$$
I_{b}=\left\{i \in I: \mathfrak{d}_{\mathfrak{m}^{\dagger}}(i)=\mathfrak{d}_{\mathfrak{m}}(i)+1\right\} .
$$

Finally, if $i \in I_{b}$ and $i^{\prime} \in I^{*}$ are such that $b\left(\Delta_{i}\right)=b\left(\Delta_{i^{\prime}}\right), \Delta_{i} \subsetneq \Delta_{i^{\prime}}$ and $\mathfrak{d}_{\mathfrak{m}}(i)=\mathfrak{d}_{\mathfrak{m}}\left(i^{\prime}\right)$, then $i^{\prime} \neq i_{\text {min }}^{*}$ and $\mathfrak{d}_{\mathfrak{m}}\left(i^{\prime}\right)+1=\mathfrak{d}_{\mathfrak{m}}\left(i^{\prime-}\right)$.

(3) $d(\mathfrak{m})=d\left(\mathfrak{m}^{\dagger}\right)$.

(4) $\mathfrak{d}_{\mathfrak{m}}$ is injective on $I^{*}$.

Remark 3.2. In general, it is not true that $\mathfrak{d}_{\mathfrak{m}}\left(i^{-}\right)=\mathfrak{d}_{\mathfrak{m}}(i)+1$ for all $i \in I^{*} \backslash\left\{i_{\text {min }}^{*}\right\}$.

Proof. Let $i \in I$. To show that $\mathfrak{d}_{\mathfrak{m}}(i) \leq \mathfrak{d}_{\mathfrak{m}^{\dagger}}(i)$ suppose that $\Delta_{i_{1}} \ll \ldots \ll \Delta_{i_{k}}$ for some $i_{1}, \ldots, i_{k} \in I$ with $i_{1}=i$. We claim that we can choose these indices so that whenever $i_{r} \in I^{*}$ for some $r$, either $r=k$ or $b\left(\Delta_{i_{r+1}}\right)>b\left(\Delta_{i_{r}}\right)+1$ or $b\left(\Delta_{i_{r+1}}\right)=b\left(\Delta_{i_{r}}\right)+1$ and $i_{r+1} \in I^{*}$. Indeed, whenever $i_{r} \in I^{*}$ with $r<k$ and $b\left(\Delta_{i_{r+1}}\right)=b\left(\Delta_{i_{r}}\right)+1$ with $i_{r+1} \notin I^{*}$ we may replace $i_{r+1}$ by the leading index $i \in I^{*}$ such that $b\left(\Delta_{i}\right)=b\left(\Delta_{i_{r+1}}\right)$. Iterating this process we will get the required property. With this extra property we have $\Delta_{i_{1}}^{*} \ll \ldots \ll \Delta_{i_{k}}^{*}$. Thus, $\mathfrak{d}_{\mathfrak{m}}(i) \leq \mathfrak{d}_{\mathfrak{m} \dagger}(i)$.

It is clear that $I_{b} \cap I^{*}=\emptyset$ and that if $i \in I_{b}$, then $\mathfrak{d}_{\mathfrak{m}^{\dagger}}(i)>\mathfrak{d}_{\mathfrak{m}}(i)$. Indeed, if $i^{\prime} \in I^{*}$ is such that $b\left(\Delta_{i}\right)=b\left(\Delta_{i^{\prime}}\right), \Delta_{i} \subsetneq \Delta_{i^{\prime}}$ and $\mathfrak{d}_{\mathfrak{m}}(i)=\mathfrak{d}_{\mathfrak{m}}\left(i^{\prime}\right)$, then $\Delta_{i}^{*}=\Delta_{i} \ll \Delta_{i^{\prime}}^{*}$ and therefore, $\mathfrak{d}_{\mathfrak{m}^{\dagger}}(i)>\mathfrak{d}_{\mathfrak{m}^{\dagger}}\left(i^{\prime}\right) \geq \mathfrak{d}_{\mathfrak{m}}\left(i^{\prime}\right)=\mathfrak{d}_{\mathfrak{m}}(i)$. Moreover, it is clear from the definition of $i_{\text {min }}^{*}$ that $i^{\prime} \neq i_{\text {min }}^{*}$ and since $i^{\prime} \in I^{*}$ we have $\Delta_{i^{\prime}} \supseteq \Delta_{i}$. We claim that $\mathfrak{d}_{\mathfrak{m}}\left(i^{\prime}\right)+1=\mathfrak{d}_{\mathfrak{m}}\left(i^{\prime-}\right)$. Clearly, $\mathfrak{d}_{\mathfrak{m}}\left(i^{\prime-}\right)>\mathfrak{d}_{\mathfrak{m}}\left(i^{\prime}\right)$. Assume on the contrary that $\mathfrak{d}_{\mathfrak{m}}\left(i^{\prime-}\right)>\mathfrak{d}_{\mathfrak{m}}\left(i^{\prime}\right)+1$. Then by Lemma 2.1(11), there exists $j \in I$ such that $\Delta_{i^{\prime}-} \ll \Delta_{j}$ and $\mathfrak{d}_{\mathfrak{m}}(j)>\mathfrak{d}_{\mathfrak{m}}\left(i^{\prime}\right)$. If $b\left(\Delta_{j}\right)=b\left(\Delta_{i^{\prime}}\right)$, then $\Delta_{j} \subsetneq \Delta_{i^{\prime}}$ by Lemma 2.1 (3), in contradiction to the fact that $i^{\prime} \in I^{*}$. Otherwise, $\Delta_{i} \ll \Delta_{j}$ and again we get a contradiction since $\mathfrak{d}_{\mathfrak{m}}(i)=\mathfrak{d}_{\mathfrak{m}}\left(i^{\prime}\right)<\mathfrak{d}_{\mathfrak{m}}(j)$.

It remains to show that $\mathfrak{d}_{\mathfrak{m}^{\dagger}}(i) \leq \mathfrak{d}_{\mathfrak{m}}(i)+1$ for all $i \in I$ with equality only if $i \in I_{\mathrm{b}}$. We prove this by induction on $\mathfrak{d}_{\mathfrak{m}^{\dagger}}(i)$.

The statement is trivial if $\mathfrak{d}_{\mathfrak{m}^{\dagger}}(i)=0$. Suppose that $\mathfrak{d}_{\mathfrak{m}^{\dagger}}(i)>0$. Then, there exists $i_{1} \in I$ such that $\mathfrak{d}_{\mathfrak{m}^{\dagger}}(i)=\mathfrak{d}_{\mathfrak{m}^{\dagger}}\left(i_{1}\right)+1$ and $\Delta_{i}^{*} \ll \Delta_{i_{1}}^{*}$. Assume first that $\Delta_{i} \nless \Delta_{i_{1}}$. Then, $i_{1} \in I^{*}, b\left(\Delta_{i}\right)=b\left(\Delta_{i_{1}}\right)$ and $\Delta_{i} \subsetneq \Delta_{i_{1}}$. Hence, by Lemma 2.1 (3), $\mathfrak{d}_{\mathfrak{m}}\left(i_{1}\right) \leq \mathfrak{d}_{\mathfrak{m}}(i)$ and therefore, by induction hypothesis $\mathfrak{d}_{\mathfrak{m}} \dagger\left(i_{1}\right)=\mathfrak{d}_{\mathfrak{m}}\left(i_{1}\right)$. Thus,

$$
\mathfrak{d}_{\mathfrak{m}^{\dagger}}(i)=\mathfrak{d}_{\mathfrak{m}^{\dagger}}\left(i_{1}\right)+1=\mathfrak{d}_{\mathfrak{m}}\left(i_{1}\right)+1 \leq \mathfrak{d}_{\mathfrak{m}}(i)+1,
$$

with equality only if $\mathfrak{d}_{\mathfrak{m}}\left(i_{1}\right)=\mathfrak{d}_{\mathfrak{m}}(i)$, which means that $i \in I_{\mathrm{b}}$.

Assume now that $\Delta_{i} \ll \Delta_{i_{1}}$. Then, $\mathfrak{d}_{\mathfrak{m}}(i) \geq \mathfrak{d}_{\mathfrak{m}}\left(i_{1}\right)+1$. It follows from the induction hypothesis that

$$
\mathfrak{d}_{\mathfrak{m}^{\dagger}}(i)=\mathfrak{d}_{\mathfrak{m}^{\dagger}}\left(i_{1}\right)+1 \leq \mathfrak{d}_{\mathfrak{m}}\left(i_{1}\right)+2 \leq \mathfrak{d}_{\mathfrak{m}}(i)+1 .
$$

Moreover, suppose that $\mathfrak{d}_{\mathfrak{m}^{\dagger}}(i)=\mathfrak{d}_{\mathfrak{m}}(i)+1$. Then, $\mathfrak{d}_{\mathfrak{m}^{\dagger}}\left(i_{1}\right)=\mathfrak{d}_{\mathfrak{m}}\left(i_{1}\right)+1$, and hence, by induction hypothesis, $i_{1} \in I_{b}$, i.e., there exists $i_{2} \in I^{*}$ such that $b\left(\Delta_{i_{2}}\right)=b\left(\Delta_{i_{1}}\right)$, $\Delta_{i_{1}} \subsetneq \Delta_{i_{2}}$ and $\mathfrak{d}_{\mathfrak{m}}\left(i_{1}\right)=\mathfrak{d}_{\mathfrak{m}}\left(i_{2}\right)$. In particular $i_{2} \neq i_{\text {min }}^{*}$. Let $i_{3}=i_{2}^{-} \in I^{*}$. Since $i_{1} \notin I^{*}$ we must have $e\left(\Delta_{i_{3}}\right) \geq e\left(\Delta_{i_{1}}\right)>e\left(\Delta_{i}\right)$. Hence, $\Delta_{i}^{*} \ll \Delta_{i_{3}}^{*}$ and therefore, $\mathfrak{d}_{\mathfrak{m}^{\dagger}}\left(i_{3}\right)<\mathfrak{d}_{\mathfrak{m}^{\dagger}}(i)$, while $\mathfrak{d}_{\mathfrak{m}}\left(i_{3}\right)=\mathfrak{d}_{\mathfrak{m}^{\dagger}}\left(i_{3}\right)$ by induction hypothesis. On the other hand, $\mathfrak{d}_{\mathfrak{m}}\left(i_{3}\right)>\mathfrak{d}_{\mathfrak{m}}\left(i_{2}\right)=\mathfrak{d}_{\mathfrak{m}}\left(i_{1}\right)=\mathfrak{d}_{\mathfrak{m}^{\dagger}}\left(i_{1}\right)-1=\mathfrak{d}_{\mathfrak{m}^{\dagger}}(i)-2$. Thus, $\mathfrak{d}_{\mathfrak{m}}\left(i_{3}\right)=\mathfrak{d}_{\mathfrak{m}^{\dagger}}(i)-1=\mathfrak{d}_{\mathfrak{m}}(i)$. On the other hand, $e\left(\Delta_{i_{3}}\right)>e\left(\Delta_{i}\right)$ and $b\left(\Delta_{i}\right) \leq$ $b\left(\Delta_{i_{1}}\right)-1=b\left(\Delta_{i_{2}}\right)-1=b\left(\Delta_{i_{3}}\right)$. We conclude that $b\left(\Delta_{i_{3}}\right)=b\left(\Delta_{i}\right)$, so that $i \in I_{b}$. This finishes the induction step. The proof of the first two parts is now complete. 
It follows from the first part that $d(\mathfrak{m}) \leq d\left(\mathfrak{m}^{\dagger}\right) \leq d(\mathfrak{m})+1$. Suppose on the contrary that $d\left(\mathfrak{m}^{\dagger}\right)=d(\mathfrak{m})+1$. Then, by the second part, there exist $i \in I$ and $i^{\prime} \in I^{*}$ such that $b\left(\Delta_{i}\right)=b\left(\Delta_{i^{\prime}}\right), \Delta_{i} \subsetneq \Delta_{i^{\prime}}$ and $\mathfrak{d}_{\mathfrak{m}}(i)=\mathfrak{d}_{\mathfrak{m}}\left(i^{\prime}\right)=d(\mathfrak{m})$. We already observed that $i^{\prime} \neq i_{\min }^{*}$. But then, $\mathfrak{d}_{\mathfrak{m}}\left(i^{\prime-}\right)>\mathfrak{d}_{\mathfrak{m}}\left(i^{\prime}\right)=d(\mathfrak{m})$, which is a contradiction.

Finally, the last part of the lemma is evident.

To go further, we study the derived multisegment of $\mathfrak{m}^{\dagger}$ and relate it to $\left(\mathfrak{m}^{\prime}\right)^{\dagger}$.

We say that an index $i \in I$ is special if $i \in I^{\natural}$ and distinguished if $i \in I^{\natural}$ or $\Delta_{i} \neq$ $\Delta_{i \vee}$. These properties depend of course on the choice of admissible enumerations of $\mathfrak{d}^{-1}(k), k=0, \ldots, d(\mathfrak{m})$ (see 2.2 .2$)$. For every $i \in I$ there exists a unique distinguished $j \in I$ such that $\Delta_{i}=\Delta_{j}$.

Proposition 3.3. Suppose that $\mathfrak{m} \neq 0$ and $\min \mathfrak{m}<\min \mathfrak{l}(\mathfrak{m})$. Then,

(1) For any $i \in I^{*}$, there exists $j \in I$ such that $b\left(\Delta_{j}\right)>b\left(\Delta_{i}\right)$ and $\mathfrak{d}_{\mathfrak{m}}(i)=$ $\mathfrak{d}_{\mathfrak{m}}(j)$. In particular, $I^{*} \cap I^{\natural}=\emptyset$ and $\mathfrak{m}$ is non-degenerate.

(2) For any $i \in I^{*}$, let $i^{\#} \in I$ be the distinguished index such that $b\left(\Delta_{i}\right)=$ $b\left(\Delta_{i \#}\right), \mathfrak{d}_{\mathfrak{m}}(i)=\mathfrak{d}_{\mathfrak{m}}\left(i^{\#}\right)$ and $e\left(\Delta_{i \#}\right)$ is minimal with respect to these properties. Then,

$$
I_{\sharp}=\left\{i^{\#}: i \in I^{*}\right\} \subseteq I^{\prime}
$$

is a set of leading indices for $\mathfrak{m}^{\prime}$. In particular,

$$
\Delta^{\circ}(\mathfrak{m})=\Delta^{\circ}\left(\mathfrak{m}^{\prime}\right) .
$$

(3) Assume (as we may) that $I^{*}$ consists of distinguished indices. In particular, $i=i^{\#}$ whenever $\Delta_{i}=\Delta_{i \#}$, for $i \in I^{*}$. Then, $\left(i_{\min }^{*}\right)^{\#}=i_{\min }^{*}$. Let $\tau$ be the permutation of the index set $I$ defined by $\tau(i)=i$ for all $i \notin I^{*} \cup I_{\sharp}$, $\tau(i)=i^{-}$for all $i \in I^{*} \backslash I_{\sharp}$, and

$$
\tau\left(i^{\#}\right)= \begin{cases}\left(i^{+}\right)^{\#} & \text { if } i \neq i_{\max }^{*} \text { and }\left(i^{+}\right)^{\#} \neq i^{+}, \\ i & \text { otherwise }\end{cases}
$$

for all $i \in I^{*}$. Then, for suitable admissible enumerations of $\mathfrak{d}_{m^{\dagger}}^{-1}(k), k=$ $0, \ldots, d(\mathfrak{m})$ we have

$$
\left(\Delta_{\tau(i)}^{*}\right)^{\prime}= \begin{cases}-\Delta_{i}^{\prime} & i \in I_{\sharp}, \\ \Delta_{i}^{\prime} & \text { otherwise, }\end{cases}
$$

for any $i \in I$. Moreover, the special indices with respect to $\mathfrak{m}$ and $\mathfrak{m}^{\dagger}$ coincide and $\tau(i)=i$ for all $i \in I^{\natural}$. In particular, $\mathfrak{l}(\mathfrak{m})=\mathfrak{l}\left(\mathfrak{m}^{\dagger}\right)$.

Proof. (1) Assume on the contrary that $i \in I^{*}$ and $b\left(\Delta_{j}\right) \leq b\left(\Delta_{i}\right)$ whenever $\mathfrak{d}_{\mathfrak{m}}(j)=\mathfrak{d}_{\mathfrak{m}}(i)$. Assume further that $b\left(\Delta_{i}\right)$ is minimal with respect to this property.

Suppose first that $i=i_{\min }^{*}$. By our assumption, we have $b\left(\Delta_{j}\right)=\min \mathfrak{m}$ whenever $\mathfrak{d}_{\mathfrak{m}}(j)=\mathfrak{d}_{\mathfrak{m}}(i)$. Let $j \in I^{\natural}$ be such that $\mathfrak{d}_{\mathfrak{m}}(j)=\mathfrak{d}_{\mathfrak{m}}(i)$. Then, $b\left(\Delta_{j}^{\prime}\right)=b\left(\Delta_{j}\right)=\min \mathfrak{m}$, in contradiction to the assumption that $\min \mathfrak{l}(\mathfrak{m})>$ $\min \mathfrak{m}$.

Suppose now that $i \neq i_{\min }^{*}$ and let $j \in I$ be any index such that $\mathfrak{d}_{\mathfrak{m}}(j)=$ $\mathfrak{d}_{\mathfrak{m}}\left(i^{-}\right)$. Then, $\mathfrak{d}_{\mathfrak{m}}(j)>\mathfrak{d}_{\mathfrak{m}}(i)$ and therefore, by Lemma 2.1)(11), there exists $i^{\prime} \in I$ such that $\Delta_{j} \ll \Delta_{i^{\prime}}$ and $\mathfrak{d}_{\mathfrak{m}}\left(i^{\prime}\right)=\mathfrak{d}_{\mathfrak{m}}(i)$. By our assumption, $b\left(\Delta_{j}\right)<$ 
$b\left(\Delta_{i^{\prime}}\right) \leq b\left(\Delta_{i}\right)$ and hence, $b\left(\Delta_{j}\right) \leq b\left(\Delta_{i^{-}}\right)$. We get a contradiction to the minimality of $i$.

It is now clear that $I^{*} \cap I^{\natural}=\emptyset$. In order to infer non-degeneracy, suppose on the contrary that $i \in I^{*}$ and $b\left(\Delta_{i}\right)=e\left(\Delta_{i}\right)$. Then, for any $j \in I$ such that $b\left(\Delta_{j}\right)>b\left(\Delta_{i}\right)$ we have $\Delta_{i} \ll \Delta_{j}$ and hence, $\mathfrak{d}_{\mathfrak{m}}(i)>\mathfrak{d}_{\mathfrak{m}}(j)$. We get a contradiction.

(2) It easily follows from the first part that $I_{\sharp} \cap I^{\natural}=\emptyset$, i.e., $I_{\sharp} \subseteq I^{\prime}$. Fix $i \in I^{*}$ and let $j=i^{\#} \in I_{\sharp}$ and $k=j^{\vee}$. Since $j \in I^{\prime}$, we have $e\left(\Delta_{j}^{\prime}\right)=e\left(\Delta_{k}\right) \leq$ $e\left(\Delta_{j}\right)$ by (2.4). Moreover, from the defining property of $i$, we get

$$
b\left(\Delta_{i}\right)=b\left(\Delta_{j}^{\prime}\right)=b\left(\Delta_{j}\right)<b\left(\Delta_{k}\right) .
$$

We split the discussion into 3 cases.

(a) Suppose first that $i=i_{\text {min }}^{*}$, so that $b\left(\Delta_{i}\right)=\min \mathfrak{m}$. Note that $\Delta_{j}=\Delta_{i}$. (If $i$ is distinguished, then $j=i$.) We claim that $\Delta_{j}^{\prime}$ is the shortest segment of $\mathfrak{m}^{\prime}$ that begins at $\min \mathfrak{m}$.

Suppose on the contrary that this is not the case. Then, there exists $l \in I^{\prime}$ such that $\Delta_{l}^{\prime} \subsetneq \Delta_{j}^{\prime} \subseteq \Delta_{i}$ and $b\left(\Delta_{l}\right)=b\left(\Delta_{i}\right)$. Since $e\left(\Delta_{l^{\vee}}\right)=$ $e\left(\Delta_{l}^{\prime}\right)$, we have $\Delta_{l \vee} \subsetneq \Delta_{i}$. On the other hand, since $i=i_{\min }^{*}$ we have $\Delta_{i} \subseteq \Delta_{l}$. If $\Delta_{i}=\Delta_{l}$, then $l$ is not distinguished (since $l \neq j$ and $\left.\Delta_{j}=\Delta_{l}\right)$ and therefore, $\Delta_{l}^{\prime}=\Delta_{i}$, which is a contradiction. Hence, $\Delta_{i} \subsetneq \Delta_{l}$. This contradicts Lemma 2.2(1).

(b) Suppose now that $i \neq i_{\text {max }}^{*}$. Set $j^{\prime}=\left(i^{+}\right)^{\#} \in I_{\sharp}$, so that $b\left(\Delta_{j^{\prime}}\right)=$ $b\left(\Delta_{i}\right)+1$. We show that $\Delta_{j}^{\prime} \ll \Delta_{j^{\prime}}^{\prime}$ but there does not exist a segment $\Delta$ of $\mathfrak{m}^{\prime}$ such that $\Delta_{j}^{\prime} \ll \Delta, b(\Delta)=b\left(\Delta_{j}^{\prime}\right)+1$ and $\Delta \subsetneq \Delta_{j^{\prime}}^{\prime}$.

Let $k^{\prime}=\left(j^{\prime}\right)^{\vee} \in I$. Since $\Delta_{i} \ll \Delta_{i^{+}}$we have

$$
\mathfrak{d}_{\mathfrak{m}}(k)=\mathfrak{d}_{\mathfrak{m}}(j)=\mathfrak{d}_{\mathfrak{m}}(i)>\mathfrak{d}_{\mathfrak{m}}\left(i^{+}\right)=\mathfrak{d}_{\mathfrak{m}}\left(j^{\prime}\right)=\mathfrak{d}_{\mathfrak{m}}\left(k^{\prime}\right)
$$

By (3.4), $b\left(\Delta_{k}\right) \geq b\left(\Delta_{j}\right)+1=b\left(\Delta_{j^{\prime}}\right)$. By Lemma 2.2(3) (applied with $\left.i=j^{\prime}, j=k\right)$, we deduce that $e\left(\Delta_{k}\right)<e\left(\Delta_{j^{\prime}}^{\prime}\right)$, which means that $\Delta_{j}^{\prime} \ll \Delta_{j^{\prime}}^{\prime}$, as claimed.

To prove the second statement, suppose on the contrary that $\Delta_{l}^{\prime}, l \in I^{\prime}$ is a segment of $\mathfrak{m}^{\prime}$ such that $\Delta_{j}^{\prime} \ll \Delta_{l}^{\prime}, b\left(\Delta_{l}^{\prime}\right)=b\left(\Delta_{j}^{\prime}\right)+1$ and $\Delta_{l}^{\prime} \subsetneq \Delta_{j^{\prime}}^{\prime}$. Thus, $b\left(\Delta_{l}\right)=b\left(\Delta_{i}\right)+1, e\left(\Delta_{j}^{\prime}\right)<e\left(\Delta_{l}^{\prime}\right)<e\left(\Delta_{k^{\prime}}\right)$ and $e\left(\Delta_{l}^{\prime}\right) \leq e\left(\Delta_{l}\right)$ (by (2.4) ). Hence, $e\left(\Delta_{j}^{\prime}\right)<e\left(\Delta_{l}\right)$. By Lemma 2.2.2) (applied with $i=j$ and $j=l)$ we conclude that $\mathfrak{d}_{\mathfrak{m}}(l)<\mathfrak{d}_{\mathfrak{m}}(j)=\mathfrak{d}_{\mathfrak{m}}(i)$.

We claim that there exists $m \in I$ such that $\Delta_{i} \ll \Delta_{m}, b\left(\Delta_{m}\right)=$ $b\left(\Delta_{i}\right)+1$ and $\mathfrak{d}_{\mathfrak{m}}(m)=\mathfrak{d}_{\mathfrak{m}}(l)$. If $\Delta_{i} \ll \Delta_{l}$, we simply take $m=l$. Otherwise, $e\left(\Delta_{l}\right) \leq e\left(\Delta_{i}\right)$ and by Lemma 2.1(11), there is $m \in I$, such that $\Delta_{i} \ll \Delta_{m}$ and $\mathfrak{d}_{\mathfrak{m}}(m)=\mathfrak{d}_{\mathfrak{m}}(l)$. In this case, $e\left(\Delta_{l}\right)<e\left(\Delta_{m}\right)$ and hence, $b\left(\Delta_{m}\right)=b\left(\Delta_{i}\right)+1$; otherwise we would have $\Delta_{l} \ll \Delta_{m}$ which is impossible.

By the definition of $i^{+}$we have $\Delta_{i^{+}} \subseteq \Delta_{m}$ and $b\left(\Delta_{i^{+}}\right)=b\left(\Delta_{m}\right)$, which implies that $\mathfrak{d}_{\mathfrak{m}}\left(i^{+}\right) \geq \mathfrak{d}_{\mathfrak{m}}(m)$. Equivalently, $\mathfrak{d}_{\mathfrak{m}}\left(k^{\prime}\right) \geq \mathfrak{d}_{\mathfrak{m}}(l)$. However, by (3.4), $b\left(\Delta_{k^{\prime}}\right)>b\left(\Delta_{j^{\prime}}\right)=b\left(\Delta_{l}\right)$ while $e\left(\Delta_{l}^{\prime}\right)<e\left(\Delta_{k^{\prime}}\right)$. We get a contradiction to Lemma 2.2(2) (applied with $i=l$ and $j=k^{\prime}$ ).

(c) Finally, suppose that $i=i_{\max }^{*}$. We show that there is no segment $\Delta$ of $\mathfrak{m}^{\prime}$ such that $\Delta_{j}^{\prime} \ll \Delta$ and $b(\Delta)=b\left(\Delta_{i}\right)+1\left(=b\left(\Delta_{j}^{\prime}\right)+1\right)$. 
The argument is similar to the previous case (but a little bit easier). Write $\Delta=\Delta_{l}^{\prime}$ with $l \in I^{\prime}$. Then, $b\left(\Delta_{l}\right)=b\left(\Delta_{i}\right)+1$ and $e\left(\Delta_{k}\right)<$ $e\left(\Delta_{l}^{\prime}\right) \leq e\left(\Delta_{l}\right)$.

As before, $\mathfrak{d}_{\mathfrak{m}}(l)<\mathfrak{d}_{\mathfrak{m}}(j)=\mathfrak{d}_{\mathfrak{m}}(i)$. By Lemma 2.1(1), there is $m \in I$, such that $\Delta_{i} \ll \Delta_{m}$ and $\mathfrak{d}_{\mathfrak{m}}(m)=\mathfrak{d}_{\mathfrak{m}}(l)$. On the other hand, since $i=i_{\max }^{*}$ we necessarily have $e\left(\Delta_{l}\right) \leq e\left(\Delta_{i}\right)$ and therefore, $e\left(\Delta_{l}\right)<$ $e\left(\Delta_{m}\right)$. We conclude that $b\left(\Delta_{m}\right)=b\left(\Delta_{i}\right)+1$, for otherwise $\Delta_{l} \ll \Delta_{m}$, in contradiction to the fact that $\mathfrak{d}_{\mathfrak{m}}(m)=\mathfrak{d}_{\mathfrak{m}}(l)$.

In conclusion, we found $m \in I$ such that $\Delta_{i} \ll \Delta_{m}$ and $b\left(\Delta_{m}\right)=$ $b\left(\Delta_{i}\right)+1$. This contradicts the assumption that $i=i_{\max }^{*}$.

The 3 cases complete the proof of this part.

(3) First note that it follows from Lemma 3.1 that if $t$ is an integer outside $\mathfrak{d}_{\mathfrak{m}}\left(I^{*}\right)$, then $\mathfrak{d}_{\mathfrak{m}}^{-1}(t)=\mathfrak{d}_{\mathfrak{m}^{\dagger}}^{-1}(t)$ and of course, $\Delta_{i}^{*}=\Delta_{i}$ for all $i \in \mathfrak{d}_{\mathfrak{m}}^{-1}(t)$. Hence, keeping the admissible enumeration on $\mathfrak{d}_{\mathfrak{m}}^{-1}(t)$ we have

$$
\left(\Delta_{i}^{*}\right)^{\prime}=\Delta_{i}^{\prime}, \text { for all } i \in I \text { with } \mathfrak{d}_{\mathfrak{m}}(i) \notin \mathfrak{d}_{\mathfrak{m}}\left(I^{*}\right) .
$$

Now, fix $i \in I^{*}$ and let $t=\mathfrak{d}_{\mathfrak{m}}(i)$. We already noted that $\left(i_{\min }^{*}\right) \#=i_{\min }^{*}$ if the latter is distinguished. Define

$$
J_{i}=\left\{j \in I: \mathfrak{d}_{\mathfrak{m}}(j)=t, b\left(\Delta_{j}\right)=b\left(\Delta_{i}\right) \text { and } \Delta_{j} \subsetneq \Delta_{i}\right\} .
$$

Recall that we fixed an admissible enumeration $\left\{i_{1}, \ldots, i_{l}\right\}$ of $\mathfrak{d}_{\mathfrak{m}}^{-1}(t)$. Let $a$ and $b$ be the indices such that $i_{a}=i$ and $i_{b}=i^{\#}$. Then, $a \leq b<l$ (by the first part) and $J_{i}=\left\{i_{r}: a<r \leq b\right\}$ since both $i$ and $i^{\#}$ are distinguished. In particular, $J_{i}=\emptyset$ if and only if $i=i^{\#}$, i.e., if and only if $i \in I_{\sharp}$. By Lemma 3.1, we have

$$
\mathfrak{d}_{\mathfrak{m}^{\dagger}}^{-1}(t)=\left(\mathfrak{d}_{\mathfrak{m}}^{-1}(t) \cup J_{i^{+}}\right) \backslash J_{i}
$$

where we set $J_{i^{+}}=\emptyset$ if $i=i_{\max }^{*}$.

Upon excising the indices of $J_{i}$ (if any) and transplanting instead the indices of $J_{i^{+}}$(if nonempty) at the same place (ordered by the admissible enumeration of $\mathfrak{d}_{\mathfrak{m}}^{-1}(t-1)$ ) we obtain an admissible enumeration $\left\{i_{1}^{\prime}, \ldots, i_{l^{\prime}}^{\prime}\right\}$ of $\mathfrak{d}_{\mathfrak{m}^{\dagger}}^{-1}(t)$ (with respect to $\mathfrak{m}^{\dagger}=\sum_{i \in I} \Delta_{i}^{*}$ ).

This recipe yields

$$
\left(\Delta_{i}^{*}\right)^{\prime}= \begin{cases}\Delta_{i^{+}}^{\prime} & \text { if } J_{i^{+}} \neq \emptyset \\ -\Delta_{i^{\prime}}^{\prime} & \text { otherwise }\end{cases}
$$

while if $J_{i+} \neq \emptyset$, then

$$
\left(\Delta_{\left(i^{+}\right)^{\#}}^{*}\right)^{\prime}={ }^{-} \Delta_{i \#}^{\prime} .
$$

For all $i^{\prime} \in \mathfrak{d}_{\mathfrak{m}^{\dagger}}^{-1}(t) \backslash\left\{i,\left(i^{+}\right)^{\#}\right\}$, we have $\left(\Delta_{i^{\prime}}^{*}\right)^{\prime}=\Delta_{i^{\prime}}^{\prime}$. In particular, $i_{l}=i_{l^{\prime}}^{\prime}$ and $\left(\Delta_{i_{l}}^{*}\right)^{\prime}=\Delta_{i_{l}}^{\prime}$. Thus, the special indices with respect to $\mathfrak{m}$ and $\mathfrak{m}^{\dagger}$ coincide and $\mathfrak{l}(\mathfrak{m})=\mathfrak{l}\left(\mathfrak{m}^{\dagger}\right)$.

It is easy to see that the relations (3.5a)-(3.5c) are equivalent to (3.3). Note that the inverse of $\tau$ is given by $\tau^{-1}(j)=j$ for all $j \notin I^{*} \cup I_{\sharp}$,

$$
\tau^{-1}(j)=\left\{\begin{array}{ll}
j^{+} & \text {if } j \neq i_{\max }^{*} \\
j^{\#} & \text { otherwise }
\end{array} \text { and }\left(j^{+}\right)^{\#} \neq j^{+} \quad j \in I^{*}\right.
$$

and

$$
\tau^{-1}\left(j^{\#}\right)=\left(j^{-}\right)^{\#}, \quad j \in I^{*} \backslash I_{\sharp} .
$$

The proposition follows. 
Corollary 3.4. For any $0 \neq \mathfrak{m} \in \mathfrak{M}$ with $\min \mathfrak{m}<\min \mathfrak{l}(\mathfrak{m})$ we have

$$
(\mathcal{K} \times \operatorname{Id})(\mathcal{M W}(\mathfrak{m}))=(\operatorname{Id} \times \mathcal{M} \mathcal{W})(\mathcal{K}(\mathfrak{m})) .
$$

In other words, $\mathfrak{l}(\mathfrak{m})=\mathfrak{l}\left(\mathfrak{m}^{\dagger}\right), \Delta^{\circ}(\mathfrak{m})=\Delta^{\circ}\left(\mathfrak{m}^{\prime}\right)$ and $\left(\mathfrak{m}^{\dagger}\right)^{\prime}=\left(\mathfrak{m}^{\prime}\right)^{\dagger}$.

Proof. We already established the relations $\mathfrak{l}(\mathfrak{m})=\mathfrak{l}\left(\mathfrak{m}^{\dagger}\right)$ and $\Delta^{\circ}(\mathfrak{m})=\Delta^{\circ}\left(\mathfrak{m}^{\prime}\right)$. Moreover,

$$
\left(\mathfrak{m}^{\dagger}\right)^{\prime}=\sum_{i \in I^{\prime}}\left(\Delta_{i}^{*}\right)^{\prime}
$$

and since $I_{\sharp}$ is a set of leading indices for $\mathfrak{m}^{\prime}$,

$$
\left(\mathfrak{m}^{\prime}\right)^{\dagger}=\sum_{i \in I_{\sharp}}-\Delta_{i}^{\prime}+\sum_{i \in I^{\prime} \backslash I_{\sharp}} \Delta_{i}^{\prime} .
$$

Therefore, the relation $\left(\mathfrak{m}^{\dagger}\right)^{\prime}=\left(\mathfrak{m}^{\prime}\right)^{\dagger}$ follows from (3.3).

\section{Representation theOREtic applications}

4.1. Basics. For the rest of the paper we fix a non-archimedean local field $F$ with normalized absolute value $|\cdot|$ and consider representations of the general linear groups $\mathrm{GL}_{n}(F), n \geq 0$. All representations are implicitly assumed to be complex and smooth.

For any segment $\Delta=[a, b] \in \operatorname{Seg}$, we write $Z(\Delta)$ and $L(\Delta)$ for the character $|\operatorname{det}|^{\frac{a+b}{2}}$ of $\mathrm{GL}_{b-a+1}(F)$ and the Steinberg representation of $\mathrm{GL}_{b-a+1}(F)$ twisted by $|\operatorname{det}|^{\frac{a+b}{2}}$, respectively.

Normalized parabolic induction will be denoted by $\times$. More precisely, if $\pi_{i}$ are representations of $\mathrm{GL}_{n_{i}}(F), i=1, \ldots, k$ and $n=n_{1}+\cdots+n_{k}$, we write

$$
\pi_{1} \times \cdots \times \pi_{k}=\operatorname{Ind}_{P_{n_{1}, \ldots, n_{k}}(F)}^{\mathrm{GL}_{n}(F)} \pi_{1} \otimes \cdots \otimes \pi_{k},
$$

where $P_{n_{1}, \ldots, n_{k}}$ is the parabolic subgroup of $\mathrm{GL}_{n}$ consisting of block upper triangular matrices with block sizes $n_{1}, \ldots, n_{k}$ and $\pi_{1} \otimes \cdots \otimes \pi_{k}$ is considered as a representation of $P_{n_{1}, \ldots, n_{k}}$ via the pull-back from $\mathrm{GL}_{n_{1}}(F) \times \cdots \times \mathrm{GL}_{n_{k}}(F)$.

Given a multisegment $\mathfrak{m} \in \mathfrak{M}$, we can write it (in possibly several ways) as $\mathfrak{m}=\sum_{i=1}^{k} \Delta_{i}$, where for any $i<j$, we have $\Delta_{i} \nless \Delta_{j}$. Then, the representations

$$
\begin{aligned}
Z(\mathfrak{m}) & =\operatorname{soc}\left(Z\left(\Delta_{1}\right) \times \cdots \times Z\left(\Delta_{k}\right)\right), \\
L(\mathfrak{m}) & =\operatorname{soc}\left(L\left(\Delta_{k}\right) \times \cdots \times L\left(\Delta_{1}\right)\right),
\end{aligned}
$$

are both irreducible and, up to equivalence, depend only on $\mathfrak{m}$. (We recall that soc stands for the socle, i.e., the sum of all irreducible subrepresentations.) The Mœglin-Waldspurger involution switches the two, namely

$$
L(\mathfrak{m}) \cong Z\left(\mathfrak{m}^{\#}\right), \quad Z(\mathfrak{m}) \cong L\left(\mathfrak{m}^{\#}\right) .
$$

In fact, the map $Z(\mathfrak{m}) \mapsto L(\mathfrak{m})$ is a specialization of a functorial duality on the category of admissible smooth representations of $\left.\mathrm{GL}_{n}, 3,25\right]$.

By the Zelevinsky classification, given integers $a_{1}, \ldots, a_{n}$, any irreducible subquotient of the representation $|\cdot|^{a_{1}} \times \cdots \times|\cdot|^{a_{n}}$ of $\mathrm{GL}_{n}(F)$ can be written in the form $Z(\mathfrak{m})$ (or alternatively, $L(\mathfrak{m})$ ) for a unique $\mathfrak{m} \in \mathfrak{M}$. 
Remark 4.1. More generally, we can fix a (not necessarily unitary) irreducible supercuspidal representation $\rho$ of $\mathrm{GL}_{d}(F)$ and consider the irreducible representations

$$
Z\left([a, b]_{\rho}\right)=\operatorname{soc}\left(\rho|\operatorname{det}|^{a} \times \cdots \times \rho|\operatorname{det}|^{b}\right), \quad L\left([a, b]_{\rho}\right)=\operatorname{soc}\left(\rho|\operatorname{det}|^{b} \times \cdots \times \rho|\operatorname{det}|^{a}\right)
$$

of $\mathrm{GL}_{(b-a+1) d}(F)$. (When $\rho$ is the trivial character of $\mathrm{GL}_{1}(F)=F^{*}$ this coincides with the previous notation.) We can then define $Z\left(\mathfrak{m}_{\rho}\right)$ and $L\left(\mathfrak{m}_{\rho}\right)$ for any multisegment $\mathfrak{m}$ as before. Theorem 4.3 and its proof will hold without change. Given irreducible supercuspidal representations $\rho_{1}, \ldots, \rho_{k}$ of $\mathrm{GL}_{d_{i}}(F)$ such that $\rho_{i} \not \rho_{j}|\operatorname{det}|^{r}$ for all $i \neq j$ and $r \in \mathbb{Z}$, and any multisegments $\mathfrak{m}_{1}, \ldots, \mathfrak{m}_{k}$, the representation $Z\left(\left(\mathfrak{m}_{1}\right)_{\rho_{1}}\right) \times \cdots \times Z\left(\left(\mathfrak{m}_{k}\right)_{\rho_{k}}\right)$ is irreducible. Moreover, by Zelevinsky classification, any irreducible representation of $\mathrm{GL}_{n}(F)$ can be written uniquely in this form (up to permuting the factors) [33. A similar statement holds for $L(\mathfrak{m})$. Therefore, for all practical purposes it is enough to deal with a single $\rho$. For concreteness we take $\rho$ to be the trivial character of $F^{*}$, but as was pointed out above this is essentially immaterial.

4.2. Suppose that $\mathfrak{l}$ is a ladder. Then, for any irreducible representation $\tau$ of $\mathrm{GL}_{n}(F)$, each of the representations $\operatorname{soc}(Z(\mathfrak{l}) \times \tau)$ and $\operatorname{soc}(\tau \times Z(\mathfrak{l}))$ is irreducible and occurs with multiplicity one in the Jordan-Hölder sequence of $Z(\mathfrak{l}) \times \tau$ [16]. Thus, for any $\mathfrak{m} \in \mathfrak{M}$ and $\mathfrak{l} \in \operatorname{Lad}$, there is a multisegment $\mathfrak{s o c}(\mathfrak{m}, \mathfrak{l}) \in \mathfrak{M}$, such that

$$
\operatorname{soc}(Z(\mathfrak{m}) \times Z(\mathfrak{l})) \cong Z(\mathfrak{s o c}(\mathfrak{m}, \mathfrak{l}))
$$

A simple recursive algorithm for the computation of $\mathfrak{s o c}(\mathfrak{m}, \mathfrak{l})$, which relies on the Mœglin-Waldspurger algorithm, is given in [ibid.] 3 We recall the result (using the notation of 93.1 .

Proposition 4.2 ([16, Proposition 6.15] and [17, Lemma 3.16]). Let $0 \neq \mathfrak{m} \in \mathfrak{M}$ and $\mathfrak{l} \in$ Lad.

(1) Suppose that $\min \mathfrak{l} \leq \min \mathfrak{m}$. Let $\Delta$ be the (unique) segment in $\mathfrak{l}$ for which $b(\Delta)=\min \mathfrak{l}$. Then,

$$
\mathfrak{s o c}(\mathfrak{m}, \mathfrak{l})=\mathfrak{s o c}(\mathfrak{m}-\mathfrak{n}, \mathfrak{l}-\Delta)+\mathfrak{n}+\Delta,
$$

where upon writing $\mathfrak{m}=\sum_{i \in I} \Delta_{i}$,

$$
\mathfrak{n}=\sum_{i \in I: b\left(\Delta_{i}\right)=b(\Delta) \text { and } e\left(\Delta_{i}\right) \leq e(\Delta)} \Delta_{i} \leq \mathfrak{m} .
$$

(2) Suppose that $\min \mathfrak{m}<\min \mathfrak{l}$. Then, $\mathfrak{s o c}(\mathfrak{m}, \mathfrak{l})$ is characterized by the condition

$$
\mathcal{M W}(\mathfrak{s o c}(\mathfrak{m}, \mathfrak{l}))=\left(\mathfrak{s o c}\left(\mathfrak{m}^{\dagger}, \mathfrak{l}\right), \Delta^{\circ}(\mathfrak{m})\right)
$$

We remark that we also have

$$
\operatorname{soc}(L(\mathfrak{l}) \times L(\mathfrak{m})) \cong L(\mathfrak{s o c}(\mathfrak{m}, \mathfrak{l})) .
$$

This can be proved by either repeating the argument of [16] or using the functorial properties of the Zelevinsky involution.

\footnotetext{
${ }^{3}$ Strictly speaking, we have to pass to the contragredient.
} 
4.3. Main result. We use the notation of $₫ 2.2$.

\section{Theorem 4.3.}

(1) For any $0 \neq \mathfrak{m} \in \mathfrak{M}$ we have

$$
\mathfrak{s o c}\left(\mathfrak{m}^{\prime}, \mathfrak{l}(\mathfrak{m})\right)=\mathfrak{m}, \quad \operatorname{soc}\left(L(\mathfrak{l}(\mathfrak{m})) \times L\left(\mathfrak{m}^{\prime}\right)\right)=L(\mathfrak{m}) .
$$

(2) For any $(\mathfrak{l}, \mathfrak{m}) \in \mathfrak{A}$ we have

$$
\mathfrak{s o c}(\mathfrak{m}, \mathfrak{l})=\mathcal{K}^{-1}(\mathfrak{l}, \mathfrak{m}), \quad \operatorname{soc}(L(\mathfrak{l}) \times L(\mathfrak{m}))=L\left(\mathcal{K}^{-1}(\mathfrak{l}, \mathfrak{m})\right) .
$$

(3) Given $0 \neq \mathfrak{m} \in \mathfrak{M}$ write $\mathcal{R S K}(\mathfrak{m})=\left(\mathfrak{l}_{1}, \ldots, \mathfrak{l}_{k}\right) \in \mathcal{L}$ and define recursively

$$
\pi_{k}=Z\left(\mathfrak{l}_{k}\right), \quad \pi_{i}=\operatorname{soc}\left(\pi_{i+1} \times Z\left(\mathfrak{l}_{i}\right)\right), \quad i=k-1, \ldots, 1 .
$$

Then, $\pi_{1} \cong Z(\mathfrak{m})$.

Similarly, letting

$$
\pi_{k}^{\prime}=L\left(\mathfrak{l}_{k}\right), \quad \pi_{i}^{\prime}=\operatorname{soc}\left(L\left(\mathfrak{l}_{i}\right) \times \pi_{i+1}^{\prime}\right), \quad i=k-1, \ldots, 1,
$$

we have $\pi_{1}^{\prime} \cong L(\mathfrak{m})$.

In particular, $Z(\mathfrak{m})$ (resp. $L(\mathfrak{m})$ ) occurs as a sub-representation of

$$
\Lambda(\mathfrak{m}):=Z\left(\mathfrak{l}_{k}\right) \times \cdots \times Z\left(\mathfrak{l}_{1}\right) \quad\left(\operatorname{resp} . \Lambda^{\prime}(\mathfrak{m}):=L\left(\mathfrak{l}_{1}\right) \times \cdots \times L\left(\mathfrak{l}_{k}\right)\right) .
$$

Proof. First note that in view of Proposition 2.4, the second part is merely a reformulation of the first part and the last part follows directly from the first two parts and the definition of $\mathcal{R} \mathcal{S} \mathcal{K}$. Thus, it suffices to prove the first part. Moreover, by the remark following Proposition 4.2 it is enough to prove the assertion

$$
\mathfrak{s o c}\left(\mathfrak{m}^{\prime}, \mathfrak{l}(\mathfrak{m})\right)=\mathfrak{m} \text {. }
$$

We argue by induction on $|\mathfrak{m}|$, using Proposition 4.2. The base of the induction (the case $\mathfrak{m}=0)$ is trivial. Suppose that $0 \neq \mathfrak{m}=\sum_{i \in I} \Delta_{i} \in \mathfrak{M}$. We separate into cases.

Suppose first that $\min \mathfrak{l}(\mathfrak{m})=\min \mathfrak{m}$. Let $i_{0} \in I$ be as in Lemma 2.6 and set

$$
\mathfrak{n}=\sum_{i \in I: \mathfrak{d}_{\mathfrak{m}}(i)=d(\mathfrak{m})} \Delta_{i} \leq \mathfrak{m} .
$$

Then, by Lemma 2.6.

$$
\mathcal{K}(\mathfrak{m}-\mathfrak{n})=\left(\mathfrak{l}(\mathfrak{m})-\Delta_{i_{0}}, \mathfrak{m}^{\prime}-\left(\mathfrak{n}-\Delta_{i_{0}}\right)\right) .
$$

Since $|\mathfrak{m}-\mathfrak{n}|<|\mathfrak{m}|$, the induction hypothesis now implies that

$$
\mathfrak{m}-\mathfrak{n}=\mathfrak{s o c}\left(\mathfrak{m}^{\prime}-\left(\mathfrak{n}-\Delta_{i_{0}}\right), \mathfrak{l}(\mathfrak{m})-\Delta_{i_{0}}\right) .
$$

It follows from the first part of Proposition 4.2 (applied to $\mathfrak{m}^{\prime}$ and $\left.\mathfrak{l}(\mathfrak{m})\right)$ and Lemma 2.6 that $\mathfrak{s o c}\left(\mathfrak{m}^{\prime}, \mathfrak{l}(\mathfrak{m})\right)=\mathfrak{m}$.

Suppose now that $\min \mathfrak{m}<\min \mathfrak{l}(\mathfrak{m})$. This implies that $\min \mathfrak{m}^{\prime}=\min \mathfrak{m}<$ $\min \mathfrak{l}(\mathfrak{m})$. Hence, we can apply the second part of Proposition 4.2 to $\mathfrak{m}^{\prime}$ and $\mathfrak{l}(\mathfrak{m})$ to obtain

$$
\mathcal{M W}\left(\mathfrak{s o c}\left(\mathfrak{m}^{\prime}, \mathfrak{l}(\mathfrak{m})\right)\right)=\left(\mathfrak{s o c}\left(\left(\mathfrak{m}^{\prime}\right)^{\dagger}, \mathfrak{l}(\mathfrak{m})\right), \Delta^{\circ}\left(\mathfrak{m}^{\prime}\right)\right)
$$

By Corollary 3.4, we get

$$
\mathcal{M W}\left(\mathfrak{s o c}\left(\mathfrak{m}^{\prime}, \mathfrak{l}(\mathfrak{m})\right)\right)=\left(\mathfrak{s o c}\left(\left(\mathfrak{m}^{\dagger}\right)^{\prime}, \mathfrak{l}\left(\mathfrak{m}^{\dagger}\right)\right), \Delta^{\circ}(\mathfrak{m})\right) .
$$

Yet, since $\left|\mathfrak{m}^{\dagger}\right|<|\mathfrak{m}|$, the induction hypothesis implies that the last expression is nothing but $\mathcal{M W}(\mathfrak{m})$. The result follows from the injectivity of the map $\mathcal{M W}$. 
Remark 4.4. In [10, the width invariant $k=k(\mathfrak{m})$ was defined for every $\mathfrak{m}=$ $\sum_{i \in I} \Delta_{i} \in \mathfrak{M}$ to be the maximal number of distinct indices $i_{1}, \ldots, i_{k} \in I$ for which $\Delta_{i_{r+1}} \subseteq \Delta_{i_{r}}$ for all $r=1, \ldots, k-1$. By standard properties of the RSK correspondence, $k(\mathfrak{m})$ is the number of rows in the tableaux of $\operatorname{RSK}^{\prime}(\mathfrak{m})$.

It was shown in [10] that if there exist $\mathfrak{l}_{1}, \ldots, \mathfrak{l}_{l} \in$ Lad such that $Z(\mathfrak{m})$ appears as a subquotient of $Z\left(\mathfrak{l}_{1}\right) \times \cdots \times Z\left(\mathfrak{l}_{l}\right)$, then $l \geq k(\mathfrak{m})$. This underlines a minimality property of $\Lambda(\mathfrak{m})$.

Remark 4.5. For $\mathfrak{m}=\sum_{i \in I}\left[a_{i}, b_{i}\right]$ let $\mathfrak{m}^{\vee}=\sum_{i \in I}\left[-b_{i},-a_{i}\right]$, so that $Z\left(\mathfrak{m}^{\vee}\right)$ is the contragredient of $Z(\mathfrak{m})$. Then, $\mathcal{R} \mathcal{S} \mathcal{K}\left(\mathfrak{m}^{\vee}\right)$ is related to $\mathcal{R} \mathcal{S} \mathcal{K}(\mathfrak{m})$ by the Schützenberger involution (modified to our conventions).

We also point out that

$$
\left(\mathfrak{m}^{\#}\right)^{\vee}=\left(\mathfrak{m}^{\vee}\right)^{\#}
$$

since $Z(\mathfrak{m})^{\vee}=Z\left(\mathfrak{m}^{\vee}\right)$ and $L\left(\mathfrak{m}^{\vee}\right)=L(\mathfrak{m})^{\vee}$.

It would be interesting to extend the second part of Theorem 4.3 to an arbitrary pair of a ladder $\mathfrak{l}$ and a multisegment $\mathfrak{m}$.

\section{Enhanced MULTisegments}

The results of the previous section stay put if we slightly extend the notion of multisegments to include empty segments of the form $[a+1, a], a \in \mathbb{Z}$ (keeping track of the parameter $a$ ).

Since the proofs are essentially the same, we will only state the results in the modified case, indicating the points where the proofs have to be adjusted.

5.1. Extended definitions. For an integer $a$, we will write $\delta_{a}=(a+1, a) \in \mathbb{Z} \times \mathbb{Z}$ and consider it as a dummy segment. As with segments, we write $b\left(\delta_{a}\right)=a+1$ and $e\left(\delta_{a}\right)=a$. We write

$$
\widetilde{\operatorname{Seg}}=\operatorname{Seg} \cup\left\{\delta_{a}: a \in \mathbb{Z}\right\}
$$

for the set of enhanced segments.

The relation $\ll$ is defined on $\widehat{\text { Seg }}$ in the same fashion as it was defined on Seg.

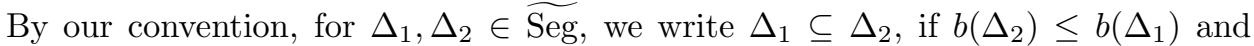
$e\left(\Delta_{1}\right) \leq e\left(\Delta_{2}\right)$.

Next, we embed the monoid $\mathbb{N}(\mathbb{Z})$ into $\mathbb{N}(\mathbb{Z} \times \mathbb{Z})$ by assigning to each $\hat{a}=$ $a_{1}+\ldots+a_{k} \in \mathbb{N}(\mathbb{Z})$ the dummy multisegment $\partial_{\hat{a}}=\delta_{a_{1}}+\ldots+\delta_{a_{k}} \in \mathbb{N}(\mathbb{Z} \times \mathbb{Z})$. We will often refer to dummy multisegments as elements of $\mathbb{N}(\mathbb{Z})$ by implicitly referring to this embedding. We write supp $\partial_{\hat{a}}$ for the underlying subset $\left\{a_{1}, \ldots, a_{k}\right\}$ of $\mathbb{Z}$ (without multiplicity).

Given a dummy multisegment $\partial$ and an integer $a \in \mathbb{Z}$, we let $n_{\partial}(a)$ denote the multiplicity of $\delta_{a}$ in $\partial$.

We will call $\widetilde{\mathfrak{M}}=\mathbb{N}(\widetilde{\mathrm{Seg}})$ the set of enhanced multisegments. Again taking $\mathbb{N}(\mathbb{Z})$ as the collection of dummy multisegments, we naturally obtain an identification

$$
\widetilde{\mathfrak{M}}=\mathfrak{M} \times \mathbb{N}(\mathbb{Z}) .
$$

Using this presentation we will denote an enhanced multisegment as $\mathfrak{m}=(\underline{\mathfrak{m}}, \partial(\mathfrak{m}))$ $\in \widetilde{\mathfrak{M}}$, where $\mathfrak{m}$ is the underlying (genuine) multisegment and $\partial(\mathfrak{m})$ is a dummy multisegment.

We may view $\widetilde{\mathfrak{M}}$ as a sub-monoid of $\mathbb{N}(\mathbb{Z} \times \mathbb{Z})$, by taking $\mathfrak{m} \mapsto \mathfrak{m}+\partial(\mathfrak{m})$. In particular, $\operatorname{RSK}^{\prime}(\mathfrak{m})=\left(P_{\mathfrak{m}}, Q_{\mathfrak{m}}\right) \in \mathcal{T}$ is well-defined, for all $\mathfrak{m} \in \widetilde{\mathfrak{M}}$. 
Since the relation $\ll$ and the dominance condition remain well-defined for enhanced segments, we may also define $\widetilde{\text { Lad }} \subseteq \widetilde{\mathfrak{M}}$ (the set of enhanced ladders) and

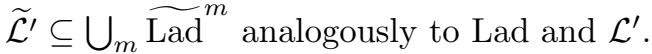

The results of $\$ 2.2 .2$ all remain valid for enhanced multisegments and, as a result, give an extension of the previously defined map $\mathcal{K}$ to

$$
\mathcal{K}: \widetilde{\mathfrak{M}} \backslash\{0\} \rightarrow \widetilde{\operatorname{Lad}} \times \widetilde{\mathfrak{M}}, \quad \mathcal{K}(\mathfrak{m})=\left(\mathfrak{l}(\mathfrak{m}), \mathfrak{m}^{\prime}\right) .
$$

Similarly, we define $\mathcal{R} \mathcal{S} \mathcal{K}: \widetilde{\mathfrak{M}} \rightarrow \widetilde{\mathcal{L}^{\prime}}$ recursively and finally obtain

$$
\operatorname{RSK}^{\prime}(\mathfrak{m})=(P(\mathcal{R S K}(\mathfrak{m})), Q(\mathcal{R S K}(\mathfrak{m})))
$$

for all $\mathfrak{m} \in \widetilde{\mathfrak{M}}$.

5.2. Enhanced results. For any enhanced multisegment $\mathfrak{m}=(\underline{\mathfrak{m}}, \partial(\mathfrak{m}))$ we write

$$
Z(\mathfrak{m})=Z(\underline{\mathfrak{m}}), \quad L(\mathfrak{m})=L(\underline{\mathfrak{m}}) .
$$

Similarly, if $\mathfrak{l}$ is an enhanced ladder and $\mathfrak{m}$ is an enhanced multisegment we write

$$
\mathfrak{s o c}(\mathfrak{l}, \mathfrak{m})=\mathfrak{s o c}(\underline{\mathfrak{l}}, \underline{\mathfrak{m}}) .
$$

In the newly defined terms, Theorem 4.3 can now be extended to the following statement.

Theorem 5.1. For any enhanced multisegment $\mathfrak{m} \in \widetilde{\mathfrak{M}}$ with non-zero $\underline{\mathfrak{m}}$, we have

$$
\mathfrak{s o c}\left(\mathfrak{m}^{\prime}, \mathfrak{l}(\mathfrak{m})\right)=\underline{\mathfrak{m}}, \quad \operatorname{soc}\left(L(\mathfrak{l}(\mathfrak{m})) \times L\left(\mathfrak{m}^{\prime}\right)\right)=L(\mathfrak{m}) .
$$

The point is that in general, $\underline{\mathfrak{m}}^{\prime} \neq \underline{\mathfrak{m}}^{\prime}$ and $\mathfrak{l}(\underline{\mathfrak{m}}) \neq \mathfrak{l}(\mathfrak{m})$. Hence, Theorem 5.1 is a genuine extension of Theorem 4.3 .

For $\mathfrak{m} \in \widetilde{\mathfrak{M}}$ with a non-zero $\underline{\mathfrak{m}}$, let us write $\mathcal{R} \mathcal{S K}(\mathfrak{m})=\left(\mathfrak{l}_{1}, \ldots, \mathfrak{l}_{k}\right) \in \widetilde{\mathcal{L}^{\prime}}$. We then form the representation

$$
\Lambda(\mathfrak{m}):=Z\left(\underline{\mathfrak{l}_{k}}\right) \times \cdots \times Z\left(\underline{\mathfrak{l}_{1}}\right) .
$$

Viewed differently, we may start from a choice of a multisegment $0 \neq \mathfrak{n} \in \mathfrak{M}$ and an auxiliary choice of a dummy multisegment $\partial \in \mathbb{N}(\mathbb{Z})$. Taking $(\mathfrak{n}, \partial) \in \widetilde{\mathfrak{M}}$, we define $\Lambda_{\partial}(\mathfrak{n}):=\Lambda(\mathfrak{n}, \partial)$.

Corollary 5.2. For any $0 \neq \mathfrak{n} \in \mathfrak{M}$ and $\partial \in \mathbb{N}(\mathbb{Z})$, the irreducible representation $Z(\mathfrak{n})$ occurs as a sub-representation of $\Lambda_{\partial}(\mathfrak{n})$.

The case $\partial=0$ amounts to Theorem 4.3.

5.2.1. On the proof of Theorem 5.1. Essentially the same arguments that appeared in the previous sections for multisegments continue to hold for the case of enhanced multisegments. In this manner, the proof of Theorem 5.1 follows the same lines as that of Theorem 4.3. Let us give some details on the necessary adjustments.

Lemma 2.6 holds for $\mathfrak{m} \in \widetilde{\mathfrak{M}} \backslash \mathbb{N}(\mathbb{Z})$, under the tweaked assumption $\min \mathfrak{l}(\mathfrak{m})=$ $\min \underline{\mathfrak{m}}$, and $d=\underline{d}(\mathfrak{m})$ being the essential depth, that is,

$$
\underline{d}(\mathfrak{m})=\max _{i \in I}\left\{\mathfrak{d}_{\mathfrak{m}}(i): \Delta_{i} \in \operatorname{Seg}\right\} .
$$

The commutativity of the two algorithms which is the culmination of $\$ 3$ requires us to extend $\mathcal{M W}$ to a map

$$
\widetilde{\mathcal{M W}}: \widetilde{\mathfrak{M}} \backslash \mathbb{N}(\mathbb{Z}) \rightarrow \widetilde{\mathfrak{M}} \times \operatorname{Seg} \quad \widetilde{\mathcal{M W}}(\mathfrak{m})=\left(\mathfrak{m}^{\dagger}, \Delta^{\circ}(\mathfrak{m})\right)
$$


as follows. Given $\mathfrak{m}=\sum_{i \in I} \Delta_{i} \in \widetilde{\mathfrak{M}}$ with $\underline{\mathfrak{m}} \neq 0$, we set $\Delta^{\circ}(\mathfrak{m})=\Delta^{\circ}(\underline{\mathfrak{m}})$ and define $\mathfrak{m}^{\dagger}=\sum_{i \in I} \Delta_{i}^{*}$ as in the multisegment case, but without discarding empty segments. In other words, if $\Delta_{i}^{*}={ }^{-} \Delta_{i}$ according to the original algorithm and $\Delta_{i}=[a, a]$, we now set $\Delta_{i}^{*}=\delta_{a} \in \widetilde{\text { Seg }}$ instead of discarding it. Note that by our convention the leading exponents $I^{*}$ are taken from $\underline{\mathfrak{m}}$ (not from the dummy part). Iterating this procedure will give rise to $\underline{\mathfrak{m}}^{\#}$ together with a dummy multisegment.

With this algorithm in mind, practically all arguments of 93 remain valid, with the notable exception of Lemma 3.1(2), whose statement should be slightly adjusted. We note that our refined definition of $\mathcal{M W}$ allows us to drop the nondegeneracy assumptions in this section. Finally, Corollary 3.4 holds for all $\mathfrak{m} \in \widetilde{\mathfrak{M}}$ with $\min \underline{\mathfrak{m}}<\min \mathfrak{l}(\mathfrak{m})$.

The arguments of $\$ 4$ can now be applied in the enhanced multisegment setting, with $\mathfrak{m}$ substituting $\mathfrak{m}$ in the required places. In the concluding part of the proof of Theorem 5.1 we use the observations that $\left(\underline{\mathfrak{m}^{\prime}}\right)^{\dagger}=\left(\mathfrak{m}^{\prime}\right)^{\dagger}=\left(\mathfrak{m}^{\dagger}\right)^{\prime}$.

5.3. Relation to standard modules. While our newly discovered family $\{\Lambda(\mathfrak{m})\}_{\mathfrak{m} \in \mathfrak{M}}$ of RSK-standard modules satisfies some intriguing properties (see next section for a further discussion), Corollary 5.2 shows that each choice of a dummy multisegment $\partial \in \mathbb{N}(\mathbb{Z})$ gives rise to yet another family $\left\{\Lambda_{\partial}(\mathfrak{m})\right\}_{\mathfrak{m} \in \mathfrak{M}}$ of representations. Fixing $\mathfrak{m} \in \mathfrak{M}$ we will show that in a suitable sense, varying $\partial$ allows us to interpolate between the standard modules à la Zelevinsky and Langlands.

Recall that for a given a multisegment $\mathfrak{m}=\sum_{i=1}^{k} \Delta_{i} \in \mathfrak{M}$, Zelevinsky constructed the standard module

$$
\zeta(\mathfrak{m})=Z\left(\Delta_{1}\right) \times \cdots \times Z\left(\Delta_{k}\right),
$$

where $\Delta_{i}$ 's are enumerated so that for any $i<j$, we have $\Delta_{i} \nless \Delta_{j}$. Similarly,

$$
\lambda(\mathfrak{m})=L\left(\Delta_{k}\right) \times \cdots \times L\left(\Delta_{1}\right),
$$

will stand for the standard module in the sense of Langlands 4

Recall further the involution $\mathfrak{m} \mapsto \mathfrak{m}^{\#}$ on $\mathfrak{M}$ (see \3.1), for which $Z(\mathfrak{m}) \cong$ $L\left(\mathfrak{m}^{\#}\right)$ and $L(\mathfrak{m}) \cong Z\left(\mathfrak{m}^{\#}\right)$. In particular, for each $\mathfrak{m} \in \mathfrak{M}, Z(\mathfrak{m})=\operatorname{soc}(\zeta(\mathfrak{m}))=$ $\operatorname{soc}\left(\lambda\left(\mathfrak{m}^{\#}\right)\right)$.

Note that viewing a segment $\Delta=[a, b] \in$ Seg as a singleton multisegment, we have $\Delta^{\#}=[a, a]+[a+1, a+1]+\ldots+[b, b] \in \operatorname{Lad}$.

Proposition 5.3. Let $\mathfrak{m}=\sum_{i \in I} \Delta_{i} \in \mathfrak{M}$ be a multisegment consisting of $r$ segments, and let $\partial \in \mathbb{N}(\mathbb{Z})$ be a dummy multisegment.

(1) Suppose that the following two conditions are satisfied.

(a) Whenever $e\left(\Delta_{j}\right)<e\left(\Delta_{i}\right)$ we have

$$
n_{\partial}\left(e\left(\Delta_{i}\right)\right) \geq n_{\partial}\left(e\left(\Delta_{j}\right)\right)+\#\left\{j^{\prime}: e\left(\Delta_{j^{\prime}}\right)=e\left(\Delta_{j}\right)\right\} .
$$

(b) For any $i \in I$ and $t$ such that $t<e\left(\Delta_{i}\right)$ we have $n_{\partial}(t) \leq n_{\partial}\left(e\left(\Delta_{i}\right)\right)$. Then, $\Lambda_{\partial}(\mathfrak{m}) \cong \zeta(\mathfrak{m})$.

(2) Suppose that $n_{\partial}(t-1) \geq n_{\partial}(t)+\#\left\{i \in I: t \in \Delta_{i}\right\}$ for all $\min \mathfrak{m}<t \leq$ $\max \mathfrak{m}\left(=\max _{i \in I} e\left(\Delta_{i}\right)\right)$ (when $\left.\mathfrak{m} \neq 0\right)$. Then, $\Lambda_{\partial}(\mathfrak{m}) \cong \lambda\left(\mathfrak{m}^{\#}\right)$.

The first part follows from the following elementary lemma by induction on $|\mathfrak{m}|+|\partial|$.

\footnotetext{
${ }^{4}$ Note that we reversed the order of factors from the usual convention in order to obtain $L(\mathfrak{m})$ as a subrepresentation, rather than a quotient.
} 
Lemma 5.4. Let $0 \neq \mathfrak{m} \in \widetilde{\mathfrak{M}}$ be an enhanced multisegment, with $\underline{\mathfrak{m}}=\sum_{i \in I} \Delta_{i}$.

(1) Suppose that $n_{\partial(\mathfrak{m})}\left(e\left(\Delta_{i}\right)\right)>0$, for all $i \in I$. Then,

(a) $\mathfrak{d}_{\mathfrak{m}}(i)=\#\left\{a>e\left(\Delta_{i}\right): n_{\partial(\mathfrak{m})}(a)>0\right\}$ for all $i \in I$. In particular, $\mathfrak{d}_{\mathfrak{m}}(i)$ depends only on $e\left(\Delta_{i}\right)$.

(b) $\mathfrak{l}(\mathfrak{m})=\sum_{a \in \operatorname{supp} \partial(\mathfrak{m})} \delta_{a}$.

(c) $\mathfrak{m}^{\prime}=\mathfrak{m}-\mathfrak{l}(\mathfrak{m})$.

(2) Suppose that $\underline{\mathfrak{m}} \neq 0$ and let $t$ be the minimum of $e\left(\Delta_{i}\right), i \in I$. Suppose that $n_{\partial(\mathfrak{m})}\left(e\left(\Delta_{i}\right)\right)>0$ for all $i$ such that $e\left(\Delta_{i}\right)>t$ but $n_{\partial(\mathfrak{m})}(a)=0$ for all $a \leq t$. Let $\Delta$ be the segment in $\mathfrak{m}$ such that $e(\Delta)=t$ and $b(\Delta)$ is maximal with respect to this property. Then,

(a) $\mathfrak{d}_{\mathfrak{m}}(i)=\#\left\{a>e\left(\Delta_{i}\right): n_{\partial(\mathfrak{m})}(a)>0\right\}$ for all $i \in I$. In particular, $\mathfrak{d}_{\mathfrak{m}}(i)$ depends only on $e\left(\Delta_{i}\right)$.

(b) $\mathfrak{l}(\mathfrak{m})=\Delta+\sum_{a \in \operatorname{supp} \partial(\mathfrak{m})} \delta_{a}$.

(c) $\mathfrak{m}^{\prime}=\mathfrak{m}-\mathfrak{l}(\mathfrak{m})$.

Indeed, if $0 \neq \mathfrak{m} \in \widetilde{\mathfrak{M}}$ satisfies the conditions of the first part of Proposition 5.3 , then either the first or the second case of Lemma 5.4 is applicable and in both cases $\mathfrak{n}=\mathfrak{m}^{\prime}$ also satisfies the conditions of the first part of Proposition 5.3. Moreover, either $\underline{\mathfrak{n}}=\underline{\mathfrak{m}}$ (in the first case) or $\mathfrak{n}$ is obtained from $\underline{\mathfrak{m}}$ by dropping the segment $\Delta$ (in the second case, with $\Delta$ as above).

For the second part of Proposition [5.3, we recall the relation $\left(\mathfrak{m}^{\#}\right)^{\vee}=\left(\mathfrak{m}^{\vee}\right)^{\#}$ for multisegments (see Remark 4.5). This means that instead of (5.1) we may consider the map

$$
\mathfrak{m} \mapsto\left(\mathfrak{m}_{\dagger}, \Delta_{\circ}(\mathfrak{m})\right),
$$

where $\mathfrak{m}_{\dagger}:=\left(\left(\mathfrak{m}^{\vee}\right)^{\dagger}\right)^{\vee}$ and $\Delta_{\circ}(\mathfrak{m}):=\left(\Delta^{\circ}\left(\mathfrak{m}^{\vee}\right)\right)^{\vee}$. Applying it iteratively will give rise to $\underline{\mathfrak{m}}^{\#}$, together with a dummy multisegment. (In fact, for genuine multisegments, this was the original description in [21.)

Hence, the second part of Proposition 5.3 follows by a simple induction from the following elementary lemma whose proof is an easy exercise (cf. [21, Remarques II.2]).

Lemma 5.5. Let $\mathfrak{m} \in \widetilde{\mathfrak{M}}$ be an enhanced multisegment with $0 \neq \underline{\mathfrak{m}}=\sum_{i \in I} \Delta_{i}$.

Suppose that $n_{\partial(\mathfrak{m})}(a)>0$ for all $\min \underline{\mathfrak{m}} \leq a<\max \underline{\mathfrak{m}}$ but $n_{\partial(\mathfrak{m})}(a)=0$ for all $a \geq \max \underline{\mathfrak{m}}$. Then,

(1) For any $i \in I, \mathfrak{d}_{\mathfrak{m}}(i)=\max \underline{\mathfrak{m}}-e\left(\Delta_{i}\right)$, if there exist $i_{0}, \ldots, i_{r}=i \in I$ with $r=\max \underline{\mathfrak{m}}-e\left(\Delta_{i}\right)$ such that $\Delta_{i}=\Delta_{i_{r}} \ll \ldots \ll \Delta_{i_{0}}$; otherwise $\mathfrak{d}_{\mathfrak{m}}(i)=\max \mathfrak{m}-e\left(\Delta_{i}\right)-1$.

(2) $\mathfrak{l}(\mathfrak{m})=\Delta_{\circ}(\mathfrak{m})^{\#}+\sum_{a: n_{\partial(\mathfrak{m})}(a)>0, a+1<b\left(\Delta_{\circ}(\mathfrak{m})\right)} \delta_{a}$.

(3) $\mathfrak{m}^{\prime}=\mathfrak{m}_{\dagger}-\sum_{a: n_{\partial(\mathfrak{m})}(a)>0} \delta_{a}$.

Once again, if $0 \neq \mathfrak{m} \in \widetilde{\mathfrak{M}}$ satisfies the condition of the second part of Proposition [5.3. then either the first case of Lemma 5.4 or Lemma 5.5] is applicable, and in both cases $\mathfrak{n}=\mathfrak{m}^{\prime}$ also satisfies the condition of the second part of Proposition 5.3. Moreover, either $\underline{\mathfrak{n}}=\underline{\mathfrak{m}}$ (in the first case) or $\underline{\mathfrak{n}}=\underline{\mathfrak{m}}_{\dagger}$ (in the second case).

Remark 5.6. While Proposition 5.3 (which is a simple combinatorial statement) and Corollary 5.2 give a new perspective on the Møglin-Waldspurger involution, they do not give a genuinely new proof of the relation $Z(\mathfrak{m})=L\left(\mathfrak{m}^{\#}\right)$, which is one 
of the main results of [21. The reason is that the techniques and ideas of [ibid.] are used in the results of [16] and hence indirectly in the proof of Theorem 5.2 .

\section{ODDS AND ENDS}

6.1. Socle irreducibility. It is natural to ask whether as in the case of the traditional standard modules, the newly constructed class of RSK-standard modules possesses the property of having a unique irreducible sub-representation. Although we are currently unable to prove this in general, we expect that this is indeed the case, namely

Conjecture 6.1. 5 Let $\mathfrak{m}$ be a multisegment and let $\Lambda(\mathfrak{m})$ be as in (4.1). Then, $\operatorname{soc}(\Lambda(\mathfrak{m}))$ is irreducible and hence (by Theorem 4.3$), Z(\mathfrak{m}) \cong \operatorname{soc}(\Lambda(\mathfrak{m}))$.

A weaker form of this conjecture would be the following

Conjecture 6.2. Suppose that $\mathcal{R S K}(\mathfrak{m})=\left(\mathfrak{l}_{1}, \ldots, \mathfrak{l}_{k}\right)$. Define recursively $\pi_{1}^{\prime}=$ $Z\left(\mathfrak{l}_{1}\right), \pi_{i}^{\prime}=\operatorname{soc}\left(Z\left(\mathfrak{l}_{i}\right) \times \pi_{i-1}^{\prime}\right), i=2, \ldots, k$. Then, $Z(\mathfrak{m}) \cong \pi_{k}^{\prime}$.

Note that Conjecture 6.2 is not a formal consequence of Theorem 4.3 since in general it is not true that $\operatorname{soc}\left(\operatorname{soc}\left(\pi_{1} \times \pi_{2}\right) \times \pi_{3}\right) \simeq \operatorname{soc}\left(\pi_{1} \times \operatorname{soc}\left(\pi_{2} \times \pi_{3}\right)\right)$, even if $\pi_{i}$ are supercuspidal. For instance, we can take $\pi_{1}=\pi_{3}$ to be the trivial character of $F^{*}$ and $\pi_{2}$ to be the absolute value on $F^{*}$.

It is tempting to attempt to prove Conjecture 6.2 by the same method as Theorem 4.3. Suppose that $\mathcal{R} \mathcal{S K}(\mathfrak{m})=\left(\mathfrak{l}_{1}, \ldots, \mathfrak{l}_{k}\right)$. Call $\mathfrak{l}_{k}$ the lowest ladder of $\mathfrak{m}$ and write $V(\mathfrak{m})=\left(\mathfrak{l}_{k},{ }^{\prime} \mathfrak{m}\right)$ where ${ }^{\prime} \mathfrak{m}=\mathcal{R} \mathcal{S} \mathcal{K}^{-1}\left(\mathfrak{l}_{1}, \ldots, \mathfrak{l}_{k-1}\right)$ (the fact that it is well defined may follow from arguments in the spirit of Appendix A). We need to show that

$$
Z(\mathfrak{m})=\operatorname{soc}\left(Z\left(\mathfrak{l}_{k}\right) \times Z\left({ }^{\prime} \mathfrak{m}\right)\right)
$$

As before, it is natural to use the recipe of [16, §6.3]. The simple case is when $\max \mathfrak{l}_{k}=\max \mathfrak{m}$. Assume that $\max \mathfrak{l}_{k}<\max \mathfrak{m}$. In this case we have to show that

$$
V\left(\mathfrak{m}^{\dagger^{\prime}}\right)=\left(\mathfrak{l}_{k},\left({ }^{\prime} \mathfrak{m}\right)^{\dagger^{\prime}}\right),
$$

where $\mathfrak{m}^{\dagger^{\prime}}$ is the analogue of $\mathfrak{m}^{\dagger}$ for the end points.

6.2. Triangular structure. For any integer $r$ and an inverted Young tableau $Y$, let $Y_{\geq r}$ be the part of $Y$ consisting of the entries that are bigger than or equal to $r$. Clearly, $Y_{\geq r}$ is also an inverted Young tableau (of possibly smaller size). Recall the dominance order on the set of Young diagrams defined by

$$
\left(\lambda_{1}, \ldots, \lambda_{k}\right) \prec\left(\lambda_{1}^{\prime}, \ldots, \lambda_{k^{\prime}}^{\prime}\right) \text { if } k \leq k^{\prime} \text { and } \sum_{i=1}^{j} \lambda_{i} \geq \sum_{i=1}^{j} \lambda_{i}^{\prime} \text { for all } j=1, \ldots, k \text {. }
$$

(We will only compare Young diagrams of the same size. In this case $\prec$ encodes the closure relation of unipotent orbits in $\mathrm{GL}_{n}(\mathbb{C})$, parameterized by partitions via the Jordan normal form.) Define a partial order on the set of inverted Young tableaux by

$$
Y \leq Y^{\prime} \quad \text { if } \operatorname{sh}\left(Y_{\geq r}\right) \prec \operatorname{sh}\left(Y_{\geq r}^{\prime}\right) \text { for all } r \in \mathbb{Z}
$$

where $\operatorname{sh}(X)$ is the shape of $X$, i.e., its underlying Young diagram. (We will only compare inverted Young tableaux whose entries coincide as multisets.) The product

\footnotetext{
${ }^{5}$ Note added in proof: Conjecture 6.1 is now proved by the first-named author (see [11]).
} 
partial order on $\mathcal{T}$ induces a partial order on $\mathcal{L}$ (which will be denoted by $\leq$ ), according to the identifications of

Conjecture 6.3. Let $\mathfrak{m}$ be a multisegment and let $\Lambda(\mathfrak{m})$ be as in (4.1). In the Grothendieck group we have

$$
[\Lambda(\mathfrak{m})]=[Z(\mathfrak{m})]+\sum_{i=1}^{l}\left[Z\left(\mathfrak{n}_{i}\right)\right],
$$

where $\mathcal{R} \mathcal{S K}\left(\mathfrak{n}_{i}\right)<\mathcal{R} \mathcal{S K}(\mathfrak{m})$ for all $i=1, \ldots, l 6$

We verified this conjecture by computer calculation for all multisegments consisting of $n$ segments with $n \leq 8$. The computation involves writing $Z\left(\mathfrak{l}_{k}\right) \times \cdots \times Z\left(\mathfrak{l}_{1}\right)$ in terms of standard modules 15 and decomposing standard modules into irreducible representations - the multiplicities are given by the value at 1 of Kazhdan-Lusztig polynomials with respect to the symmetric group $S_{n}$.

We do not know whether the partial order on $\mathfrak{M}$ (or on the symmetric group for that matter) given by $\mathfrak{m}_{1} \leq \mathfrak{m}_{2} \Longleftrightarrow \mathcal{R S K}\left(\mathfrak{m}_{1}\right) \leq \mathcal{R} \mathcal{S K}\left(\mathfrak{m}_{2}\right)$ has already been considered in the literature. Likewise, we are so far unaware of a befitting geometric interpretation or a simpler combinatorial description of this partial order.

Let $\mathcal{R}_{n}, n \geq 0$ be the Grothendieck groups of $\mathrm{GL}_{n}(F), n \geq 0$ and let $\mathcal{R}^{\prime}$ be the subgroup of $\oplus_{n \geq 0} \mathcal{R}_{n}$ generated by $[Z(\mathfrak{m})], \mathfrak{m} \in \mathfrak{M}$.

Conjecture 6.3 would imply the following weaker conjecture.

Conjecture 6.4. The classes of RSK-standard representations

$$
\Lambda(\mathfrak{m}), \mathfrak{m} \in \mathfrak{M}
$$

form a $\mathbb{Z}$-basis for $\mathcal{R}^{\prime}$.

6.3. Relation to the DRS basis. Recall that $\mathcal{R}^{\prime}$, equipped with the parabolic induction product, becomes a commutative ring, which is freely generated by the

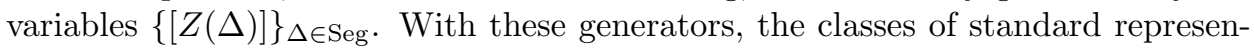
tations become the monomial basis for $\mathcal{R}^{\prime}$ (as a $\mathbb{Z}$-module).

Let $\bar{a}, \bar{b} \subseteq \mathbb{Z}$ be two given subsets of integers. Let $\mathfrak{M}_{\bar{a}, \bar{b}}$ be the collection of multisegments $\mathfrak{m}$, for which the begin points (resp. end points) of all segments comprising $\mathfrak{m}$ belong to $\bar{a}$ (resp. $\bar{b}$ ). Let $\mathcal{R}_{\bar{a}, \bar{b}}$ be the subgroup of $\mathcal{R}^{\prime}$ generated by $[Z(\mathfrak{m})], \mathfrak{m} \in \mathfrak{M}_{\bar{a}, \bar{b}}$. In other words, $\mathcal{R}_{\bar{a}, \bar{b}}$ is the subring of $\mathcal{R}^{\prime}$ generated by the classes of $Z([a, b])$ where $a \in \bar{a}$ and $b \in \bar{b}$ and $a \leq b$. By the properties of $\mathrm{RSK}^{\prime}$, we have $[\Lambda(\mathfrak{m})] \in \mathcal{R}_{\bar{a}, \bar{b}}$, for all $\mathfrak{m} \in \mathfrak{M}_{\bar{a}, \bar{b}}$. Clearly, Conjecture 6.4 is equivalent to the statement that $\Lambda(\mathfrak{m}), \mathfrak{m} \in \mathfrak{M}_{\bar{a}, \bar{b}}$, is a $\mathbb{Z}$-basis for $\mathcal{R}_{\bar{a}, \bar{b}}$, for all $\bar{a}, \bar{b}$.

Now, assume that $\bar{a}, \bar{b}$ are such that $a \leq b$, for every $a \in \bar{a}$ and $b \in \bar{b}$ (the so-called saturated case - cf. Remark 2.5). Then, $\mathcal{R}_{\bar{a}, \bar{b}}$ can be identified with the coordinate ring of the space of matrices of size $|\bar{a}| \times|\bar{b}|$. Bases for such polynomial rings were considered in [6, 7] in the context of combinatorial invariant theory. Our class of RSK-standard modules inside the rings $\mathcal{R}_{\bar{a}, \bar{b}}$ becomes precisely the bitableaux basis considered in [ibid.]. Thus, with the above identifications in place, we obtain a weak form of Conjecture 6.4

\footnotetext{
${ }^{6}$ The $\mathfrak{n}_{i}$ 's are not necessarily distinct. Also, not all $\mathfrak{n}$ 's with $\mathcal{R S K}(\mathfrak{n})<\mathcal{R} \mathcal{S K}(\mathfrak{m})$ necessarily occur.
} 
Proposition 6.5 (cf. [6, 7, 29]). For $\bar{a}, \bar{b} \subseteq \mathbb{Z}$ in the saturated case, the classes

$$
[\Lambda(\mathfrak{m})], \mathfrak{m} \in \mathfrak{M}_{\bar{a}, \bar{b}},
$$

form a $\mathbb{Z}$-basis for $\mathcal{R}_{\bar{a}, \bar{b}}$.

Proof. Under our assumptions, $\mathcal{R} \mathcal{S} \mathcal{K}$ gives a bijection between $\mathfrak{M}_{\bar{a}, \bar{b}}$ and the set of pairs of tableaux $(P, Q)$ in $\mathcal{T}$, for which $P$ (resp. $Q$ ) is filled by numbers from $\bar{a}$ (resp. $\bar{b}$ ) (see Remark 2.5). Thus, it is enough to prove that the set of elements

$$
\mathcal{B}=\left\{\left[Z\left(\mathfrak{l}_{1}\right)\right] \cdot \ldots \cdot\left[Z\left(\mathfrak{l}_{m}\right)\right]:\left(\mathfrak{l}_{1}, \ldots, \mathfrak{l}_{m}\right) \in \mathcal{L}^{\prime}, \mathfrak{l}_{i} \in \mathfrak{M}_{\bar{a}, \bar{b}}\right\}
$$

gives a basis for $\mathcal{R}_{\bar{a}, \bar{b}}$.

For a ladder $\mathfrak{l}=\sum_{i=1}^{k}\left[a_{i}, b_{i}\right]$, we have the following determinantal identity [15]

$$
[Z(\mathfrak{l})]=\sum_{\sigma \in S_{k}} \epsilon(\sigma) \prod_{i=1}^{k}\left[Z\left(\left[a_{i}, b_{\sigma(i)}\right]\right)\right],
$$

as elements of $\mathcal{R}^{\prime}$. Here, $\epsilon(\sigma)$ is the sign of the permutation $\sigma$.

Identifying $\left[Z\left(\left[a_{i}, b_{j}\right]\right)\right]$ with variables in the polynomial ring $\mathcal{R}_{\bar{a}, \bar{b}}$, we see that our construction of $\mathcal{B}$ coincides with the construction of the basis in [6, $\S 4]$.

\section{Appendix A. Multisegments, MW, And RSK BY MarK Shimozond7}

This appendix contains two main combinatorial results. The first result, Proposition A.9 describes the effect of the map $\mathcal{M W}$ on the cRSK tableau pair. The second result, Theorem A.13, is a characterization of the image of the set of multisegments under RSK expressed in terms of the right and left key tableau construction of Lascoux and Schützenberger, applied to the RSK tableau pair. The complete proofs will appear in a separate publication.

In this appendix, to conform with the bulk of the combinatorics literature, semistandard Young tableaux are used; in contrast, in the main part of this article, inverted Young tableaux are used; see Remark A.2.

A.1. Partitions and skew shapes. Let $\mathbb{Y}$ be Young's lattice of partitions. The diagram $D(\lambda)$ of $\lambda \in \mathbb{Y}$ is the set $D(\lambda)=\left\{(i, j) \in \mathbb{Z}_{>0}^{2} \mid 1 \leq j \leq \lambda_{i}\right\}$. The $(i, j)$-th box is in the $i$-th row and $j$-th column with matrix-style indexing. The transpose or conjugate partition $\lambda^{t} \in \mathbb{Y}$ is defined by $(i, j) \in D(\lambda)$ if and only if $(j, i) \in D\left(\lambda^{t}\right)$. The notation $\mu \subseteq \lambda$ means $D(\mu) \subseteq D(\lambda)$. If $\mu \subseteq \lambda$, let $\lambda / \mu:=D(\lambda) \backslash D(\mu)$; this is called a skew shape. The skew shape refers to this set difference of boxes, and can be achieved by various pairs $(\lambda, \mu)$. A skew shape is normal (resp. antinormal) if it has a unique northwestmost (resp. southeastmost) corner. A skew shape is a horizontal (resp. vertical) strip if it has at most one box in each column (resp. row). Let $\mathcal{H}$ (resp. $\mathcal{V}$ ) denote the set of horizontal (resp. vertical) strips and let $\mathcal{H}_{r}$ (resp. $\mathcal{V}_{r}$ ) be the set of horizontal (resp. vertical) strips of size $r$.

\footnotetext{
${ }^{7}$ Department of Mathematics, Virginia Polytechnic Institute and State University; mshimo@math.vt.edu.
} 
A.2. Skew tableaux. In this section a semistandard tableau of shape $\lambda / \mu$ is a function $T: \lambda / \mu \rightarrow \mathbb{Z}_{>0}$ which is weakly increasing within rows going from left to right and strictly decreasing from bottom to top. Let $\mathrm{Tab}_{\lambda / \mu}$ denote the set of semistandard tableaux of shape $\lambda / \mu$. A tableau (resp. antitableau) is a semistandard skew tableau of normal (resp. antinormal) shape. More precisely, by a skew tableau we mean a translation equivalence class of such maps. The (row-reading) word of a skew tableau $T$ is defined by word $(T)=\cdots u^{(2)} u^{(1)}$ where $u^{(i)}$ is the word obtained by reading the $i$-th row of $T$ from left to right. Using the row reading word a skew tableau may be regarded as a word. A tableau (resp. antitableau) word is a word of the form word $(T)$ where $T$ is a tableau (resp. antitableau).

A.3. Knuth equivalence. Let $\equiv$ be Knuth's equivalence relation on the set of words $\mathbb{A}^{*}$ with symbols in the totally ordered set $\mathbb{A}=\{1<2<\cdots<n\}[13$. It is the transitive closure of relations $u x z y v \equiv u z x y v$ for $x \leq y<z$ and $u y x z v \equiv u y z x v$ for $x<y \leq z$ where $x, y, z \in \mathbb{A}$ and $u, v \in \mathbb{A}^{*}$.

Proposition A.1 ([13]). Every Knuth class in $\mathbb{A}^{*}$ contains a unique tableau word and a unique antitableau word.

Denote by $\mathbb{P}(u)$ the unique tableau whose word is Knuth equivalent to $u$. This is the Schensted $P$-tableau. For a tableau $T$ denote by $T \searrow$ the unique antitableau whose word is Knuth equivalent to the word of $T$. Then, $\mathbb{P}(u) \searrow$ is the unique antitableau word that is Knuth equivalent to $u$.

Remark A.2.

(1) An inverted Young tableau of $₫ 2.2$ is the same thing as an antitableau except written a different way: they are mapped to each other by reflection across a northeast to southwest antidiagonal. See further A.6.

(2) Passing from a tableau $T$ to its antitableau $T^{\searrow}$ is a nontrivial computation. Given the word of $T^{\searrow}$, taking its reverse complement with respect to some ambient alphabet containing all appearing entries, directly gives the word of a tableau known as the Schützenberger involution of $T$.

A.4. Pieri bijections. A row word (resp. column word) is a weakly increasing (resp. strictly decreasing) word. Let Row (resp. Col) denote the set of row (resp. column) words and Row $r$ (resp. $\mathrm{Col}_{r}$ ) those of length $r$.

Proposition A.3 ([18, Théorème 2.10]). Let $\mu \in \mathbb{Y}$ and $r \in \mathbb{Z}_{\geq 0}$. There are bijections

$$
\begin{gathered}
\operatorname{Row}_{r} \times \operatorname{Tab}_{\mu} \cong \bigsqcup_{\substack{\lambda \in \mathbb{Y} \\
\lambda / \mu \in \mathcal{H}_{r}}} \operatorname{Tab}_{\lambda} \\
\operatorname{Col}_{r} \times \operatorname{Tab}_{\mu} \cong \bigsqcup_{\substack{\lambda \in \mathbb{Y} \\
\lambda / \mu \in \mathcal{V}_{r}}} \operatorname{Tab}_{\lambda}
\end{gathered}
$$$$
(u, T) \mapsto \mathbb{P}(u T),
$$$$
(u, T) \mapsto \mathbb{P}(u T),
$$$$
\operatorname{Tab}_{\mu} \times \operatorname{Row}_{r} \cong \bigsqcup_{\substack{\lambda \in \mathbb{Y} \\ \lambda / \mu \in \mathcal{H}_{r}}} \operatorname{Tab}_{\lambda}
$$$$
(T, u) \mapsto \mathbb{P}(T u),
$$$$
\operatorname{Tab}_{\mu} \times \operatorname{Col}_{r} \cong \bigsqcup_{\substack{\lambda \in \mathbb{Y} \\ \lambda / \mu \in \mathcal{V}_{r}}} \operatorname{Tab}_{\lambda}
$$$$
(T, u) \mapsto \mathbb{P}(T u)
$$ 
Example A.4. We illustrate how to compute the inverse of the bijection (A.2). Let $\lambda=(4,3,3,2,1)$ and $\mu=(3,2,2,1,1)$. Let $U$ be the unique skew standard tableau of shape $\lambda / \mu \in \mathcal{V}$, in which the boxes are labeled in increasing value from top to bottom. Pictured below is a tableau $X$ of shape $\lambda$. We perform successive Schensted reverse column insertions starting at the boxes of $U$ starting with the bottommost. Each reverse insertion produces an additional single entry factored to the left: the bumping path along which entries shift to the left is depicted in green.

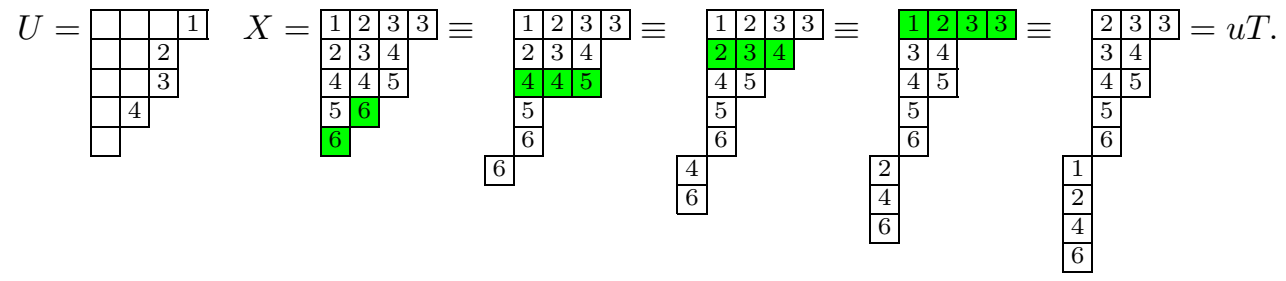

Example A.5. We illustrate how to compute the inverse of the bijection (A.4). We use the same $\lambda, \mu$, and $U$ as before but a different tableau $Y$. We perform successive Schensted reverse row insertions starting at the boxes of $U$ starting with the bottommost. Each reverse insertion produces an additional single entry factored to the right: the bumping path along which entries shift upwards is depicted in green.

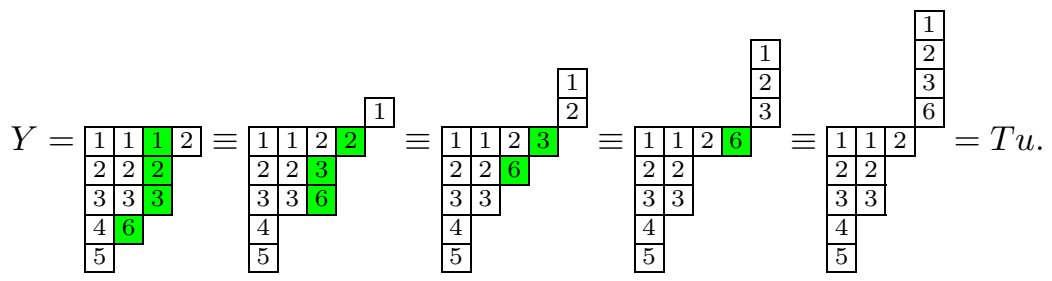

A.5. Column RSK correspondence. Let Row* be the set of infinite sequences of row words $u^{\bullet}=\left(\cdots, u^{(2)}, u^{(1)}\right)$ with $u^{(i)} \in$ Row, only finitely many of which are nonempty.

Given $u^{\bullet} \in$ Row $^{*}$ let $P_{0}=\emptyset$ be the empty tableau and for $j \geq 1$ let $P_{j}=$ $\mathbb{P}\left(u^{(j)} P_{j-1}\right)$. Let $\mathbb{P}\left(u^{\bullet}\right)=\mathbb{P}\left(\cdots u^{(2)} u^{(1)}\right)$, the Schensted $P$-tableau of the juxtaposition of the row words $u^{(i)}$. Note that shape $\left(P_{j}\right) / \operatorname{shape}\left(P_{j-1}\right) \in \mathcal{H}$ by Proposition A.3 A.1 . This sequence of horizontal strips defines a semistandard tableau $\mathbb{Q}\left(u^{\bullet}\right)$ of the same shape as $\mathbb{P}\left(u^{\bullet}\right)$ in which the boxes of the horizontal strip shape $\left(P_{j}\right) / \operatorname{shape}\left(P_{j-1}\right)$ are filled with $j$ 's for all $j$. This yields a bijection we denote by cRSK, the column insertion Robinson-Schensted-Knuth correspondence:

$$
\text { Row }^{*} \stackrel{\mathrm{cRSK}}{\longrightarrow} \bigsqcup_{\lambda} \mathrm{Tab}_{\lambda} \times \mathrm{Tab}_{\lambda}
$$

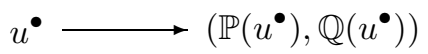


Example A.6. Let $u^{\bullet}=(\cdots, 56,34,233,122,12,1)$. We have $P_{0}$ empty,

$$
\begin{aligned}
& P_{1}=1 \\
& P_{2}=\begin{array}{l|l|}
1 & 1 \\
\hline 2 &
\end{array} \\
& P_{3}=\begin{array}{|l|l|l|l|}
\hline 1 & 1 & 1 & 2 \\
\hline 2 & 2 &
\end{array} \\
& P_{4}=\begin{array}{|l|l|l|l|}
\hline 1 & 1 & 1 & 2 \\
\hline 2 & 2 & 2 \\
\hline 3 & 3 & \multicolumn{3}{|l}{} \\
\hline
\end{array} \\
& P_{5}=\begin{array}{|l|l|l|l|}
\hline 1 & 1 & 1 & 2 \\
\hline 2 & 2 & 2 & \\
\hline 3 & 3 & 3 \\
\hline 4 & &
\end{array} \\
& \mathbb{P}\left(u^{\bullet}\right)=P_{6}=\begin{array}{|l|l|l|l|}
\hline 1 & 1 & 1 & 2 \\
\hline 2 & 2 & 2 & \\
\hline 3 & 3 & 3 \\
\hline 4 & 6 & \multicolumn{1}{|l}{} \\
\hline 5 & \multicolumn{3}{|l}{}
\end{array} \\
& \mathbb{Q}\left(u^{\bullet}\right)=\begin{array}{|l|l|l|l|}
\hline 1 & 2 & 3 & 3 \\
\hline 2 & 3 & 4 & \\
\hline 4 & 4 & 5 \\
\hline 5 & 6 & \\
\hline 6 & \multicolumn{1}{|l}{}
\end{array}
\end{aligned}
$$

A.6. Comparison with multisegment notation. Without loss of generality in this discussion we only consider segments which are intervals of positive integers. Define the injective map $\iota: \mathfrak{M} \rightarrow$ Row $^{*}$ such that $\iota(\mathfrak{m})=u^{\bullet}=\left(\cdots u^{(2)}, u^{(1)}\right)$ where the word $u^{(j)}$ contains the value $i$ the same number of times the multisegment $[i, j]$ appears in $\mathfrak{m}$. The image of $\iota$ is the set of flagged tuples of row words, those such that all values appearing in $u^{(i)}$ are less than or equal to $i$.

Let $\nearrow$ be the reflection across an antidiagonal. There is an involutive bijection inv : $\mathrm{Tab}_{\lambda} \rightarrow \mathrm{ITab}_{\lambda^{\prime}}$ from the set of semistandard tableaux of shape $\lambda$ to the set ITab $\lambda_{\lambda^{\prime}}$ of inverted tableaux of the conjugate or transposed shape $\lambda^{\prime}$, defined by $\operatorname{inv}(T)=\swarrow(T \searrow)$. That is, first take the antitableau and then flip.

$$
\begin{array}{|l|l|l|l|l|l|}
\hline 1 & 1 & 4 \\
\hline 2 & 3 \\
\hline
\end{array} \rightarrow \begin{array}{|l|l|l|}
\hline & 1 \\
\hline 2 & 3 & 4 \\
\hline
\end{array} \rightarrow \begin{array}{|l|l|}
\hline & 1 \\
\hline 2 & 1
\end{array}
$$

The following diagram commutes:

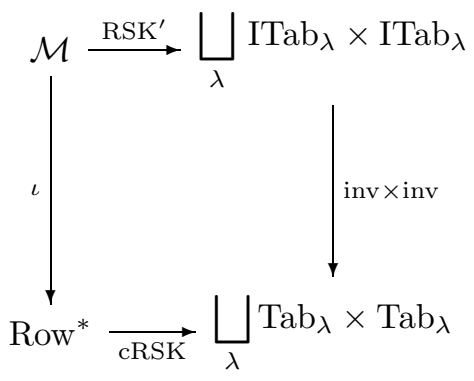

Let $\operatorname{RSK}^{\prime}(\mathfrak{m})=\left(P_{\mathfrak{m}}, Q_{\mathfrak{m}}\right)$ and $\mathcal{K}(\mathfrak{m})=\left(\mathfrak{l}(\mathfrak{m}), \mathfrak{m}^{\prime}\right)$. Then,

- $\mathfrak{l}(\mathfrak{m})$ is the ladder $\left[a_{1}, b_{1}\right]+\left[a_{2}, b_{2}\right]+\cdots+\left[a_{r}, b_{r}\right]$ where $a_{1} a_{2} \cdots a_{r}$ and $b_{1} b_{2} \cdots b_{r}$ are the first rows of $P_{\mathfrak{m}}$ and $Q_{\mathfrak{m}}$ respectively.

- $\mathfrak{m}^{\prime}$ is uniquely specified by the condition that $P_{\mathfrak{m}^{\prime}}$ and $Q_{\mathfrak{m}^{\prime}}$ are obtained from $P_{\mathfrak{m}}$ and $Q_{\mathfrak{m}}$ by removing their respective first rows.

Let $\iota(\mathfrak{m})=u^{\bullet}$, so that by the commutativity of the above diagram, $\mathbb{P}\left(u^{\bullet}\right)=$ $\operatorname{inv}\left(P_{\mathfrak{m}}\right)$ and $\mathbb{Q}\left(u^{\bullet}\right)=\operatorname{inv}\left(Q_{\mathfrak{m}}\right)$. Consider the map Row ${ }^{*} \rightarrow$ Lad $\times$ Row $^{*}$ mapping $u^{\bullet}$ to $\left(\mathfrak{l}\left(u^{\bullet}\right),\left(u^{\bullet}\right)^{\prime}\right)$ commuting the diagram

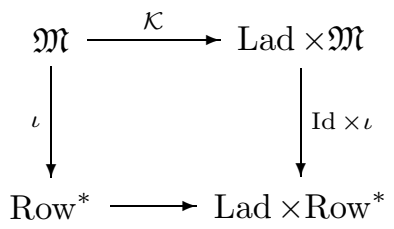


By definition, the ladder $\mathfrak{l}\left(u^{\bullet}\right)$ is obtained by the pair of column words coming from the rightmost rows of the antitableaux $\mathbb{P}\left(u^{\bullet}\right)^{\searrow}$ and $\mathbb{Q}\left(u^{\bullet}\right) \searrow$. Rather than computing the full antitableaux there is a more efficient way to compute $\mathfrak{l}\left(u^{\bullet}\right)$ and $\left(u^{\bullet}\right)^{\prime}$. Let $\mathbb{P}\left(u^{\bullet}\right) \mapsto(T, u)$ be the application of the inverse of the bijection (A.4) for $\lambda=\operatorname{shape}\left(\mathbb{P}\left(u^{\bullet}\right)\right)$ and $\mu$ obtained by removing the first column of $\lambda$. Similarly $\mathbb{Q}\left(u^{\bullet}\right) \mapsto(U, v)$. Then, $(u, v)$ is the pair of column words defining $\mathfrak{l}\left(u^{\bullet}\right)$. Moreover $\left(u^{\bullet}\right)^{\prime}$ is uniquely defined by the property that $\mathbb{P}\left(\left(u^{\bullet}\right)^{\prime}\right)=T$ and $\mathbb{Q}\left(\left(u^{\bullet}\right)^{\prime}\right)=U$.

Remark A.7. There is a version of $\mathcal{K}$ which, instead of producing the last columns of the antitableaux of $\mathbb{P}\left(u^{\bullet}\right)$ and $\mathbb{Q}\left(u^{\bullet}\right)$, produces the first columns of $\mathbb{P}\left(u^{\bullet}\right)$ and $\mathbb{Q}\left(u^{\bullet}\right)$.

A.7. The map $\mathcal{M W}$ and cRSK. Let $u^{\bullet} \in$ Row $^{*}$ be upper triangular and nonempty with corresponding multisegment $\mathfrak{m}$. Let $u^{\bullet \dagger} \in$ Row $^{*}$ correspond to $\mathfrak{m}^{\dagger}$ and let $\Delta^{\circ}\left(u^{\bullet}\right)=\Delta^{\circ}(\mathfrak{m})$.

Given a nonempty word $u$ and $m \in \mathbb{Z}_{>0}$, let $U_{m}(u)$ be the word obtained by scanning $u$ from right to left, selecting the rightmost copy of $m$ in $u$, then the next $m+1$ to its left, the next $m+2$ to its left, and so on, until the left end of $u$ is encountered, and then replacing each selected letter $i$ by $i+1$. It can be shown that $U_{m}$ can be defined on $\mathrm{Tab}_{\lambda / \mu}$ via the row reading word and on and on Row ${ }^{*}$ via the map $u^{\bullet} \mapsto \cdots u^{(2)} u^{(1)}$. Given $u^{\bullet} \in$ Row $^{*}$ nonempty, let $m=\min \left(u^{\bullet}\right)$ be the minimum value among the words in $u^{\bullet}$. Denote by $\hat{k}=\hat{k}\left(u^{\bullet}\right) \in \mathbb{Z}_{\geq 0}$ the maximum index such that $m+j \in u^{(m+j)}$ for all $0 \leq j<\hat{k}$. Define $\hat{U}\left(u^{\bullet}\right) \in$ Row $^{*}$ by removing a copy of $m+j$ from $u^{(m+j)}$ for all $0 \leq j<\hat{k}$.

We briefly define the algorithm $\mathcal{M W}$ on $u^{\bullet}$ and describe its effect on the cRSK tableau pair. Let $\min \left(u^{\bullet}\right)$ be the minimum value among the words in $u^{\bullet}$. We first consider $\hat{U}\left(u^{\bullet}\right)$ with $m=\min \left(u^{\bullet}\right)$ and $\hat{k}=\hat{k}\left(u^{\bullet}\right)$. Then, $u^{\bullet \dagger}=U_{m+\hat{k}}\left(u^{\bullet}\right)$. Let $k=k_{m}\left(u^{\bullet}\right)$ be the sum of $\hat{k}$ and the number of values incremented by $U_{m+\hat{k}}$. By definition $\Delta^{\circ}\left(u^{\bullet}\right)=[m, m+k-1]$.

Example A.8. With $u^{\bullet}$ as in Example A.6 we have $\hat{U}\left(u^{\bullet}\right)=(\cdots, 56,34,233,122$, $1, \emptyset), m=1, \hat{k}=2, u^{\bullet \dagger}=U_{3}\left(\hat{U}\left(u^{\bullet}\right)\right)=(\cdots, 66,35,234,122,1, \emptyset)$ and $k=5$.

Given a tableau $T$ and a subtableau $S$, both of partition shape, let $T-S$ be the skew tableau obtained by removing $S$ from $T$. For $a \leq b$ let $C_{a}^{b}$ be the column word $b(b-1) \cdots(a+1) a$.

Proposition A.9. Let $\emptyset \neq u^{\bullet} \in$ Row $^{*}, m=\min \left(u^{\bullet}\right),(P, Q)=\left(\mathbb{P}\left(u^{\bullet}\right), \mathbb{Q}\left(u^{\bullet}\right)\right)$, $m=\min (P)$, and $\left(P^{\dagger}, Q^{\dagger}\right)=\left(\mathbb{P}\left(u^{\bullet \dagger}\right), \mathbb{Q}\left(u^{\bullet \dagger}\right)\right)$.

(1) $\hat{k}\left(u^{\bullet}\right)$ is the maximum $\hat{k} \in \mathbb{Z}_{\geq 0}$ such that $C=C_{m}^{m+\hat{k}-1}$ is contained in the first column of $Q$.

(2) The value $k$ in the definition of $u^{\bullet \dagger}$ equals the maximum $k$ such that $C_{m}^{m+k-1}$ is contained in the first column of $P$.

(3) We have

$$
Q^{\dagger}=\mathbb{P}(Q-C)
$$

In particular shape $(Q) / \operatorname{shape}\left(Q^{\dagger}\right) \in \mathcal{V}_{\hat{k}}$.

(4) Let $(T, c) \in \operatorname{Tab}_{\text {shape }\left(Q^{\dagger}\right)} \times \operatorname{Col}_{\hat{k}}$ be the unique pair of Proposition A.3 such that $\mathbb{P}(T c)=P$. Then, $T=\mathbb{P}\left(\hat{U}\left(u^{\bullet}\right)\right), c=C$, and $P^{\dagger}=U_{m+\hat{k}}(T)$. 


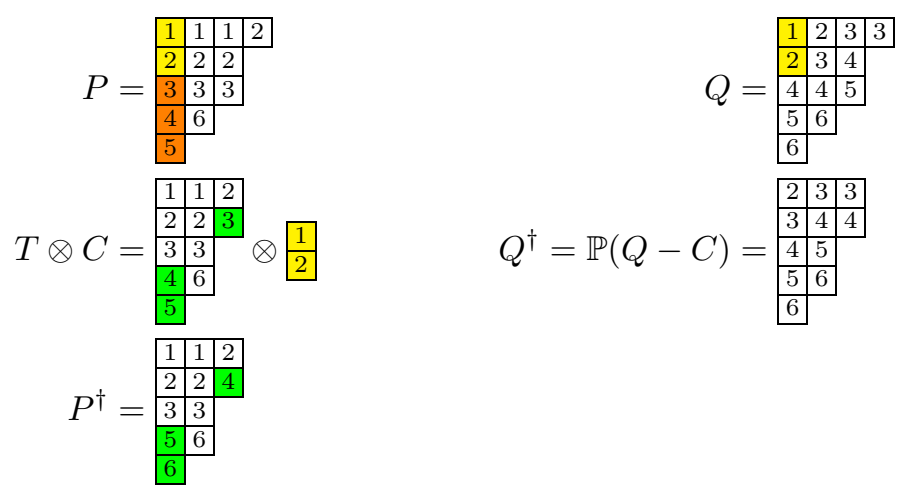

Figure 1. The computation of $Q^{\dagger}$ and $P^{\dagger}$ from $Q$ and $P$

Example A.10. With $u^{\bullet}$ as in Example A.6 $\left(P^{\dagger}, Q^{\dagger}\right)$ are given in Figure 1 computed as in Proposition A.9.

A.8. Key tableaux. Say that a tableau is a key tableau if it has the property that any of its columns, viewed as a subset, contains any column to its right.

Consider a word with symbols in the set $\{1,2, \ldots, n\}$. The weight of a word $u$ is the sequence $\operatorname{wt}(u)=\left(m_{1}(u), m_{2}(u), \ldots, m_{n}(u)\right) \in \mathbb{Z}_{\geq 0}^{n}$ where $m_{j}(u)$ is the multiplicity of the entry $j$ in $u$.

Given $\beta \in \mathbb{Z}_{\geq 0}^{n}$ let $\beta^{+}$be the unique partition in the orbit $S_{n} \cdot \beta$ and let $w_{\beta} \in S_{n}$ be the shortest element such that $w_{\beta}\left(\beta^{+}\right)=\beta$. The key tableau $\mathbb{K}_{\beta}$ of weight $\beta$ is the unique tableau of shape $\beta^{+}$and weight $\beta$. Given a fixed partition $\lambda$ the orbit $S_{n} \cdot \lambda$ is a poset: $\alpha \leq \beta$ if $w_{\alpha} \leq w_{\beta}$ in Bruhat order.

A.9. Left and right keys; image of RSK'. Recall horizontal and vertical strips from A.1. Say that a vertical (resp. horizontal) strip is $\lambda$-removable if it has the form $\lambda / \mu$ for some $\mu$.

Say that a vertical strip of size $r$ is grounded if it contains a box in each of the first $r$ rows. It is obvious that for a given $r$ a partition has at most one grounded removable vertical strip of size $r$. This strip exists if and only if $\lambda$ has a column of size $r$.

Let $P$ be a tableau of shape $\lambda$. Let $L=\lambda_{1}$ be the number of columns of $\lambda$. For each column size $r=\lambda_{j}^{t}$ of $\lambda$, let $\mu \subseteq \lambda$ be the unique partition such that $\lambda / \mu \in \mathcal{V}_{r}$ is grounded. By Proposition A.3 there is a unique pair $(u, T) \in \mathrm{Col}_{r} \times \mathrm{Tab}_{\mu}$ (resp. $(T, u) \in \mathrm{Tab}_{\mu} \times \mathrm{Col}_{r}$ such that $\mathbb{P}(u T)=P($ resp. $\mathbb{P}(T u)=P)$. Denote the word $u$ by $c_{j}^{-}(P)\left(\right.$ resp. $\left.c_{j}^{+}(P)\right)$.

Lemma A.11. Considering column words as subsets, we have $c_{j}^{-}(P) \supseteq c_{j+1}^{-}(P)$ and $c_{j}^{+}(P) \supseteq c_{j+1}^{+}(P)$ for all $j$.

The left key $K_{-}(P)$ (resp. right key $K_{+}(P)$ ) of $P$ is by definition the tableau of the same shape as $P$ whose $j$-th column is given by the column word $c_{j}^{-}(P)$ $\left(\right.$ resp. $\left.c_{j}^{+}(P)\right)$ for all $j$. By Lemma A.11 $K_{-}(P)$ and $K_{+}(P)$ are key tableaux and in particular semistandard.

Example A.12. For the tableaux $(P, Q)=\left(\mathbb{P}\left(u^{\bullet}\right), \mathbb{Q}\left(u^{\bullet}\right)\right)$ from Example A.6 the factorizations defining $c_{J}^{+}(P)$ and $c_{j}^{-}(Q)$ are given as follows. See Example A.5 for 
the method of producing the 4-box right column factor from $P$ and Example A.4 for how to obtain the 4-box left column factor from $Q$.

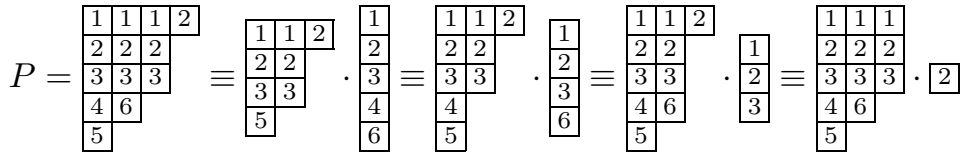

$$
\begin{aligned}
& K_{+}(P)=\begin{array}{ll|l|l|l}
\hline 1 & 1 & 1 & 2 \\
\hline 2 & 2 & 2 & \\
3 & 3 & 3 \\
\hline 4 & 6 & \\
\cline { 1 - 2 } 6 &
\end{array} \quad \operatorname{wt}\left(K_{+}(P)\right)=(3,4,3,1,0,2)
\end{aligned}
$$

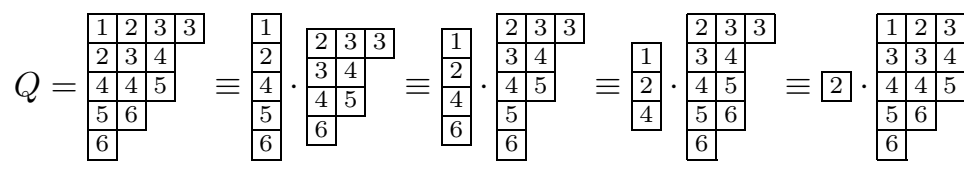

$$
\begin{aligned}
& K_{-}(Q)=\begin{array}{ll|l|l}
\hline & 1 & 1 & 2 \\
\hline 2 & 2 & 2 & \\
\hline 4 & 4 & 4 \\
\hline 5 & 6 & \\
\hline 6 &
\end{array} \quad \operatorname{wt}\left(K_{-}(Q)\right)=(3,4,0,3,1,2) .
\end{aligned}
$$

For $\alpha=\operatorname{wt}\left(K_{+}(P)\right)=(3,4,3,1,0,2)$ we have $w_{\alpha}=s_{1} s_{5} s_{4}$ and for $\beta=\operatorname{wt}\left(K_{-}(Q)\right)$ $=(3,4,0,3,1,2)$ we have $w_{\beta}=s_{1} s_{3} s_{5} s_{4} s_{5}$. We see that $w_{\alpha} \leq w_{\beta}$.

Theorem A.13. Let $u^{\bullet} \in$ Row $^{*}$. Then, $u^{\bullet}$ is flagged (in the image of a multisegment under $\iota)$ if and only if $\operatorname{wt}\left(K_{+}\left(\mathbb{P}\left(u^{\bullet}\right)\right)\right) \leq \operatorname{wt}\left(K_{-}\left(\mathbb{Q}\left(u^{\bullet}\right)\right)\right)$.

This theorem can be proved using the ideas of 22 combined with the combinatorics of the crystal graphs of Demazure modules in highest weight modules for $U_{q}\left(\mathfrak{s l}_{n}\right) 8$

Remark A.14. A similar condition works for enhanced multisegments; the flagged condition for $u^{\bullet}$ is changed to requiring that all values in $u^{(i)}$ be at most $i+1$, and that instead of Bruhat-comparing the weights $\alpha$ and $\beta$ of the right key of $\mathbb{P}\left(u^{\bullet}\right)$ and the left key of $\mathbb{Q}\left(u^{\bullet}\right)$, one uses the weight $\left(0, \beta_{1}, \beta_{2}, \ldots\right)$ instead of $\beta$.

A.10. Theorem A.13 in terms closer to inverted tableaux. We wish to state the condition of Theorem A.13 in terms of the inverted tableau pair $\left(P_{\mathfrak{m}}, Q_{\mathfrak{m}}\right)$. The first transformation is to apply $\swarrow$ so instead the condition is on a pair of antitableaux. It suffices to define left and right keys for an antitableau. By definition the left key and right key apply to a tableau. Since each Knuth class contains a unique tableau word and antitableau word, it makes sense to talk about the left and right keys of an antitableau. This suffices to describe the required condition implicitly.

However, since the map between a tableau and its antitableau is nontrivial, we wish to give a more direct way to compute the left and right keys, directly from the antitableau. For this it suffices to compute the column factors $c_{j}^{ \pm}$of an antitableau.

We will do this by example, using anti-analogues of the methods given in Examples A.4 and A.5

Example A.15. Consider the tableau $X$ of shape $\lambda=(4,3,3,2,1)$ from Example A.4. Suppose instead of $X$ we are given its antitableau. It is pictured in Figure 2.

${ }^{8}$ Note added in proof: see [1] Theorem 1.13]. 


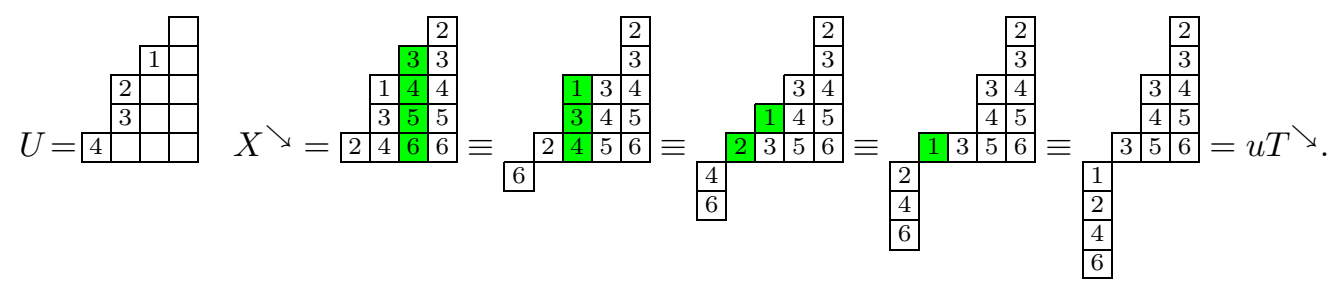

Figure 2. Computing the left key of an antitableau

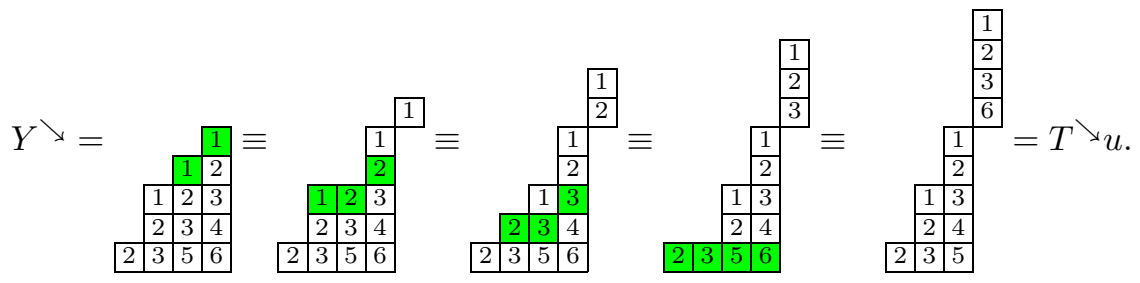

Figure 3. Computing the right key of an antitableau

Let us compute the column $c_{2}^{-}\left(X^{\searrow}\right)$, which has size 4 since $\lambda_{2}^{t}=4$. Let $\mu \subseteq \lambda$ be the unique partition such that $\lambda / \mu$ is the grounded $\lambda$-removable vertical strip of size 4 . We make a skew tableau $U$ of the anti-shape of $\lambda / \mu$ filled with the numbers 1 through 4 from top to bottom.

We now perform a sequence of successive internal row insertions on $X \searrow$ at the boxes of $U$ starting with the box labeled 1 (cf. [24]). The bumping paths, which push entries downward, are in green. Example A.4 produced a factorization $X \equiv u T$ : the current computation produces the factorization $X^{\searrow} \equiv u T^{\searrow}$.

Example A.16. Example A.5 computed a factorization $Y \equiv T u$. We illustrate a factorization $Y^{\searrow} \equiv T^{\searrow} u$. The antitableau $Y^{\searrow}$ is pictured in Figure 3. Using the same tableau $U$ as in Example A.15 we apply successive internal column insertions on $Y^{\searrow}$ at the boxes of $U$ in the same order as before. The bumping paths push to the right.

\section{ACKNOWLEDGMENTS}

We are very grateful to Mark Shimozono for writing the appendix to this paper.

Part of this work was carried out while the authors participated in the monthlong activity "On the Langlands Program: Endoscopy and Beyond" at the Institute for Mathematical Sciences of the National University of Singapore, Dec. 2018 - Jan. 2019. The first-named author and Mark Shimozono took part in the thematic trimester program on representation theory in the Institut Henri Poincaré, Paris, Jan. 2020, where the fruitful conditions of the venue led to the addition of Appendix A. We would like to thank the IMS, the IHP and the organizers of the two programs for their warm hospitality. Special thanks are due to Bernard Leclerc for an illuminating discussion on the nature of his related work [20]. Finally, we are extremely grateful to the referee for carefully reading the paper and providing many suggestions for improvement. 


\section{REFERENCES}

[1] Tomoyuki Arakawa and Takeshi Suzuki, Duality between $\mathfrak{s l}_{n}(\mathbf{C})$ and the degenerate affine Hecke algebra, J. Algebra 209 (1998), no. 1, 288-304, DOI 10.1006/jabr.1998.7530. MR:1652134

[2] Dan Barbasch and Dan Ciubotaru, Ladder representations of $G L\left(n, \mathbb{Q}_{p}\right)$, Representations of reductive groups, Progr. Math., vol. 312, Birkhäuser/Springer, Cham, 2015, pp. 117-137. MR.3495794

[3] Joseph Bernstein, Roman Bezrukavnikov, and David Kazhdan, Deligne-Lusztig duality and wonderful compactification, Selecta Math. (N.S.) 24 (2018), no. 1, 7-20, DOI 10.1007/s00029018-0391-5. MR.3769724

[4] Jonathan Brundan and Alexander Kleshchev, Blocks of cyclotomic Hecke algebras and Khovanov-Lauda algebras, Invent. Math. 178 (2009), no. 3, 451-484, DOI 10.1007/s00222009-0204-8. MR 2551762

[5] Vyjayanthi Chari and Andrew Pressley, Quantum affine algebras and affine Hecke algebras, Pacific J. Math. 174 (1996), no. 2, 295-326. MR1405590

[6] J. Désarménien, Joseph P. S. Kung, and Gian-Carlo Rota, Invariant theory, Young bitableaux, and combinatorics, Advances in Math. 27 (1978), no. 1, 63-92, DOI 10.1016/00018708(78)90077-4. MR 485944

[7] Peter Doubilet, Gian-Carlo Rota, and Joel Stein, On the foundations of combinatorial theory. IX. Combinatorial methods in invariant theory, Studies in Appl. Math. 53 (1974), 185-216, DOI 10.1002/sapm1974533185. MR498650

[8] William Fulton, Young tableaux, London Mathematical Society Student Texts, vol. 35, Cambridge University Press, Cambridge, 1997. With applications to representation theory and geometry. MR 1464693

[9] Maxim Gurevich, Quantum invariants for decomposition problems in type A rings of representations, J. Combin. Theory Ser. A 180 (2021), 105431, 46, DOI 10.1016/j.jcta.2021.105431. MR 4213657

[10] Maxim Gurevich, Decomposition rules for the ring of representations of non-Archimedean $G L_{n}$, Int. Math. Res. Not. IMRN 20 (2020), 6815-6855, DOI 10.1093/imrn/rnz006. MR 4172670

[11] Maxim Gurevich, Simple modules for quiver Hecke algebras and the Robinson-SchenstedKnuth correspondence. arXiv:2106.03120, 2021.

[12] Anthony Henderson, Nilpotent orbits of linear and cyclic quivers and Kazhdan-Lusztig polynomials of type A, Represent. Theory 11 (2007), 95-121, DOI 10.1090/S1088-4165-07-003172. MR2320806

[13] Donald E. Knuth, Permutations, matrices, and generalized Young tableaux, Pacific J. Math. 34 (1970), 709-727. MR 272654

[14] Arno Kret and Erez Lapid, Jacquet modules of ladder representations (English, with English and French summaries), C. R. Math. Acad. Sci. Paris 350 (2012), no. 21-22, 937-940, DOI 10.1016/j.crma.2012.10.014. MR.2996769

[15] Erez Lapid and Alberto Mínguez, On a determinantal formula of Tadić, Amer. J. Math. 136 (2014), no. 1, 111-142. MR.3163355

[16] Erez Lapid and Alberto Mínguez, On parabolic induction on inner forms of the general linear group over a non-archimedean local field, Selecta Math. (N.S.) 22 (2016), no. 4, 2347-2400. MR.3573961

[17] Erez Lapid and Alberto Mínguez, Geometric conditions for $\square$-irreducibility of certain representations of the general linear group over a non-archimedean local field, Adv. Math. 339 (2018), 113-190, DOI 10.1016/j.aim.2018.09.027. MR3866895

[18] Alain Lascoux and Marcel-P. Schützenberger, Le monö̈de plaxique (French, with Italian summary), Noncommutative structures in algebra and geometric combinatorics (Naples, 1978), Quad. "Ricerca Sci.", vol. 109, CNR, Rome, 1981, pp. 129-156. MR646486

[19] Bernard Leclerc, Maxim Nazarov, and Jean-Yves Thibon, Induced representations of affine Hecke algebras and canonical bases of quantum groups, Studies in memory of Issai Schur (Chevaleret/Rehovot, 2000), Progr. Math., vol. 210, Birkhäuser Boston, Boston, MA, 2003, pp. 115-153. MR.1985725 
[20] Bernard Leclerc and Jean-Yves Thibon, The Robinson-Schensted correspondence, crystal bases, and the quantum straightening at $q=0$, Electron. J. Combin. 3 (1996), no. 2, Research Paper 11, approx. 24. The Foata Festschrift. MR1392496

[21] C. Møglin and J.-L. Waldspurger, Sur l'involution de Zelevinski (French), J. Reine Angew. Math. 372 (1986), 136-177, DOI 10.1515/crll.1986.372.136. MR.863522

[22] Victor Reiner and Mark Shimozono, Key polynomials and a flagged Littlewood-Richardson rule, J. Combin. Theory Ser. A 70 (1995), no. 1, 107-143, DOI 10.1016/0097-3165(95)90083-7. MR 1324004

[23] Daniele Rosso, Classic and mirabolic Robinson-Schensted-Knuth correspondence for partial flags, Canad. J. Math. 64 (2012), no. 5, 1090-1121, DOI 10.4153/CJM-2011-071-7. MR2979579

[24] Bruce E. Sagan and Richard P. Stanley, Robinson-Schensted algorithms for skew tableaux, J. Combin. Theory Ser. A 55 (1990), no. 2, 161-193, DOI 10.1016/0097-3165(90)90066-6. MR 1075706

[25] Peter Schneider and Ulrich Stuhler, Representation theory and sheaves on the Bruhat-Tits building, Inst. Hautes Études Sci. Publ. Math. 85 (1997), 97-191. MR1471867

[26] Nicolas Spaltenstein, Classes unipotentes et sous-groupes de Borel (French), Lecture Notes in Mathematics, vol. 946, Springer-Verlag, Berlin-New York, 1982. MR672610

[27] Richard P. Stanley, Enumerative combinatorics. Vol. 2, Cambridge Studies in Advanced Mathematics, vol. 62, Cambridge University Press, Cambridge, 1999. With a foreword by Gian-Carlo Rota and appendix 1 by Sergey Fomin, DOI 10.1017/CBO9780511609589. MR 1676282

[28] Robert Steinberg, An occurrence of the Robinson-Schensted correspondence, J. Algebra 113 (1988), no. 2, 523-528, DOI 10.1016/0021-8693(88)90177-9. MR.929778

[29] Richard G. Swan, On the straightening law for minors of a matrix. arXiv:1605.06696, 2016.

[30] G. Viennot, Une forme géométrique de la correspondance de Robinson-Schensted (French), Combinatoire et représentation du groupe symétrique (Actes Table Ronde CNRS, Univ. Louis-Pasteur Strasbourg, Strasbourg, 1976), Springer, Berlin, 1977, pp. 29-58. Lecture Notes in Math., Vol. 579. MR0470059

[31] A. V. Zelevinskiǔ, The p-adic analogue of the Kazhdan-Lusztig conjecture (Russian), Funktsional. Anal. i Prilozhen. 15 (1981), no. 2, 9-21, 96. MR617466

[32] A. V. Zelevinskiǔ, Two remarks on graded nilpotent classes (Russian), Uspekhi Mat. Nauk 40 (1985), no. 1(241), 199-200. MR783619

[33] A. V. Zelevinsky, Induced representations of reductive $\mathfrak{p}$-adic groups. II. On irreducible representations of $G L(n)$, Ann. Sci. École Norm. Sup. (4) 13 (1980), no. 2, 165-210. MR584084 ISRAEL

Department of Mathematics, Technion - Israel Institute of Technology, Haifa,

Email address: maxg@technion.ac.il

Department of Mathematics, Weizmann Institute of Science, Rehovot, Israel

Email address: erez.m.lapid@gmail.com 